\title{
STATIC ECOLOGICAL SYSTEM MEASURES
}

\author{
HUSEYIN COSKUN*
}

A Holistic Analysis of Compartmental Systems

\begin{abstract}
A new mathematical method for the static analysis of ecological systems has recently been developed by the author and was presented in a separate article. Based on this methodology, multiple new ecological system measures and indices of matrix, vector, and scalar types are systematically introduced in the present paper. These mathematical system analysis tools are quantitative ecological indicators that monitor the flow distribution and storage organization, quantify the direct, indirect, acyclic, cycling, and transfer (diact) effects and utilities of one compartment-directly or indirectly — on another, and determine the residence times and compartmental activity levels. Major flow- and stock-related concepts and quantities of the current static network analyses are also integrated with the proposed measures and indices within this novel and unifying mathematical framework. This comprehensive framework enables a holistic view and analysis of static ecological systems. A quantitative technique for the classification and characterization of interspecific interactions and the determination of their strength within food webs is also developed. The proposed methodology allows for both input- and output-oriented analyses of ecological networks. The holistic perspective of the proposed methodology is extended further from the input-oriented to the output-oriented analysis. The proposed system measures and indices, thus, extract detailed information about ecosystems's characteristics, as well as their functions, properties, behaviors, and various other system attributes that are potentially hidden in and even obscured by data.
\end{abstract}

Key words. complex systems theory, ecological network analysis, compartmental systems, system and subsystem partitioning, subsystem scaling, diact flows and storages, diact effect measures and indices, diact utility measures and indices, diact residence times, food webs, interspecific interactions, input-output economics, socioeconomic systems, epidemiology, infectious diseases, toxicology, pharmacokinetics, neural networks, chemical and biological systems, control theory, information theory, information diffusion, social networks, computer networks, malware propagation, traffic flow

AMS subject classifications. 34A34, 35A24, 37N25, 37N40, 70G60, 91B74, 92B20, 92C42, 92D30, 92D40, 93C15

1. Introduction. Environmental problems have been a common topic of scholarly conversation for decades. As environmental issues persist and proliferate, the language and methods through which these problems are examined also evolve. Although traditional ecology has been used effectively in dealing with a variety of complex environmental problems, the field remains largely descriptive in nature. It has yet to arrive at a formal theory and methodology for analyzing the complex relationships between organisms and their environment or man and nature.

Ecosystems are natural systems made up of living and non-living components. Ecosystem ecology deals with interactions between species and their physical environment. More specifically, this interdisciplinary science studies the flows of energy and matter between the biotic and abiotic components of ecosystems based on conservation principles. Ecosystem ecology plays an important role in understanding current global environmental problems and determining how local mechanisms interact with these problems. Enhancements in dealing with environmental issues will ultimately depend on advances in such basic sciences. Mathematical theories and modeling are at the forefront of continued endeavors leading to a more formalistic and theoretical ecological science devoted to the discovery of basic scientific laws. Compartmental models are generally used for mathematical abstractions of ecological systems where the compartments represent ecosystem components. Within this

\footnotetext{
*Department of Mathematics, University of Georgia, Athens, GA 30602 (hcoskun@uga.edu).
} 
mathematical framework, system measures and indices are then formulated to serve as quantitative ecological indicators.

Ecological models are widely analyzed in literature, but current methodologies are developed for special cases, such as linear systems and static models. Ecological networks and complexity in living systems are analyzed, for example, at steady state in the context of information theory and thermodynamics [55, 24, 56, 57], as well as the hierarchy theory [1]. Along parallel research lines, building on economic inputoutput analysis [29, 30] introduced into ecology by [22], another static approach called the environ theory has also been developed over recent decades based on conservation principles $[40,35]$. Several software developments computerize these static methods $[58,8,17,27,50,5]$.

Despite the fact that major environmental problems of the day involve change, dynamic analysis of nonlinear systems has remained a long-standing, open problem. A mathematical theory and method recently developed by $[13,11,12]$ potentially addresses the mismatch between current static and computational methods and applied ecological needs. This comprehensive mathematical method is introduced for the dynamic analysis of nonlinear ecological systems. A separate article by [14] details the static version of this methodology. The proposed methodology is based on the novel analytical and explicit, mutually exclusive and exhaustive system and subsystem partitioning methodologies. While the proposed system partitioning yields the subthroughflows and substorages to determine the distribution of environmental inputs and the organization of associated storages individually and separately within the system, the subsystem partitioning yields the transient and the dynamic direct, indirect, acyclic, cycling, and transfer (diact) flows and storages to determine the distribution of intercompartmental flows and the organization of associated storages within the subsystems. Consequently, the fate of environmental inputs and arbitrary intercompartmental system flows, as well as the associated storages generated by these inputs and flows can be tracked individually and separately within the system. In effect, the proposed methodology brings a novel, formal, deterministic, complex system theory to the service of urgent environmental problems of the day.

The proposed dynamic methodology constructs a base for the development of new mathematical system analysis tools as quantitative ecosystem indicators. Based on this dynamic methodology, the measures and indices for the dynamic diact effects, utilities, exposures, and residence times, as well as the corresponding system efficiencies, stress, and resilience are formulated analytically by [11]. The main goal of the present manuscript is to introduce the static versions of these dynamic system analysis tools for the static ecosystem analysis.

The indirect effects in ecosystems have long been a well-established empirical fact $[38,51,61,37,36,60]$. The theoretical ecological explorations of the concept began as early as the 1970s $[26,44,19,33]$. Although the indirect effects have been a topic of scholarly conversation for the past five decades, the indirect flows and storages have never been formulated before. The indirect effects are particularly important for the characterization and classification of interspecific interactions within food webs. The classification through direct relationships alone can turn out to be incorrect without holistically considering the entire network of interactions. Community ecology qualitatively describes interspecific interactions using network topology. On the other hand, for complex networks, such characterization becomes increasingly difficult, if possible at all $[62,37,36,25,7]$. A parametric characterization is proposed in the literature, however, it has some disadvantages due to the method formulation as detailed in Section 2.10 [54, 44, 18, 16, 53]. A novel quantitative technique for the characteri- 
zation and classification of the main interspecific interaction types, and, notably, for the determination of their strength is also developed in the present manuscript. This quantitative technique that uses system flows and storages for the characterization of interspecific interactions sets up a bridge between two main branches of ecology: ecosystem ecology and community ecology.

The indirect effects are one of the multiple mathematical system analysis tools developed in the present manuscript as quantitative ecological indicators. In fact, not only the indirect effects, but also the measures and indices of matrix, vector, and scalar types for the diact effects, utilities, and residence times are systematically formulated for the first time in literature. The proposed measures and indices monitor the flow distribution and storage organization, quantify the effect or utility of one compartment directly or indirectly on another or itself, and determine the residence times and compartmental activity levels within ecosystems. As a result, these measures quantify ecosystems' characteristics, including their functions, features, and properties, and various other system attributes that are potentially hidden in and even obscured by data. They ultimately enable the characterization and classification of ecosystems, precise analyses of system structure and behavior, as well as a detailed understanding of the dynamics of individual system compartments. The proposed methodology, therefore, leads to a holistic analysis of ecosystems and serves as a quantitative platform for testing empirical hypotheses, ecological inferences, and, potentially, theoretical developments.

The proposed methodology is a comprehensive approach in the sense that the new measures and indices outlined above and major flow- and stock-related results of ecological mathematics are combined and integrated effectively within this novel and unifying mathematical framework. Through this methodology, unique relationships among some current static measures are unveiled, and corrections in some existing formulations are also suggested. The proposed methodology allows for analysis of static systems based on both their environmental inputs and outputs. The output-oriented analysis backtracks outputs instead of forward tracking inputs as the input-oriented analysis requires. The holistic perspective of the proposed methodology is extended further from the input-oriented to the output-oriented analysis. In summary, this is the first manuscript in literature that develops a holistic mathematical methodology for analysis of ecological networks.

The proposed measures and indices are applicable to any compartmental system regardless of its nature, whether naturogenic or anthropogenic. It can be used, for example, to analyze mass or energy transfers between species of different trophic levels in a complex network or along a given food chain of a food web as mentioned above and detailed in Sections 2.10 [3]. The proposed method can also be used for the analysis of models designed for material flows in industry or the dynamics of the terrestrial carbon cycle $[2,48]$. Although the motivating applications are ecological and environmental for this paper, the applicability of the proposed method extends to other realms, such as economics, pharmacology, epidemiology, chemical reaction kinetics, neural networks, biomedical systems, and information science -in fact, wherever compartmental models of conserved quantities can be constructed.

The proposed methodology is applied to two ecosystem models in Section 3 to illustrate the efficiency and wide applicability of the proposed measures and indices. The first case study concerns energy transfer within the Cone Spring ecosystem, and the second examines nitrogen transfer within the Neuse River Estuary ecosystem. The energy and nitrogen flow distributions and storage organizations within the systems, as well as the transient and diact flows and storages are already presented for these 
two ecosystem models by [14]. In the present manuscript, the measures and indices for the diact effects, utilities, and residence times are presented for the models. Ecologically, the diact effect measures and indices quantify the diact influence of the species or compartments, in terms of energy or nitrogen transfer, on the other species or compartments within the systems. The diact utility measures and indices then determine the relative diact effects of the species or compartments on each other. Moreover, the residence time matrix is used to ascertain the compartmental activity levels. The results for both input- and output-oriented analyses are presented in the case studies for a holistic view of both the Cone Spring and Neuse River Estuary ecosystems.

It is demonstrated, in particular, that the proposed methodology can be used for the classification of ecosystems based on the dominance of one of the diact effects. The seasonality in Neuse River Estuary ecosystem is also captured through the diact effect and residence time analysis. Is is hypothesized that, possibly due to the slower biological activities during the winters, the transfer flows fall short of completing the nitrogen cycle and, therefore, the acyclic flows dominates the cycling flows within this ecosystem during the winters. A gradual increase is also ascertained in net nitrogen inflow transmitted directly or indirectly from all system compartments to the phytoplankton, which potentially explains the harmful algal blooms that the Neuse River Estuary has been experiencing [10]. The quantitative technique for the characterization and classification of main types of interspecific interactions proposed in the present paper is applied to the Cone Spring ecosystem model. Importantly, it is demonstrated that the ten percent energy transfer law from one to the next trophic level introduced by [31] is not an accurate estimate, at least for these ecosystems [14].

This paper is organized as follows: the underlying dynamic and static mathematical methodologies developed by $[11,14]$ are summarized in Sections 2.1-2.2, the holistic perspective of the proposed method is detailed in Sec. 2.4, the ecosystem measures and indices are formulated systematically in Sections 2.5-2.9, results and examples are provided in Section 3, and discussion and conclusions follow in Sections 4 and 5.

2. Methods. A new mathematical theory and method has been recently developed for dynamic analysis of nonlinear compartmental systems by $[13,11]$. The proposed theory is based on the dynamic system and subsystem partitioning methodologies that determine the distribution of environmental inputs and intercompartmental flows and the organization of the associated storages generated by these inputs and flows individually and separately within the systems. The proposed methodology, therefore, as a whole, yields the decomposition of all system flows and storages to the utmost level. The dynamic methodology and its static version that has been recently developed by [14] are summarized below. The terminology and notations used in this paper are adopted from [13].

Based on the proposed methodology, multiple measures and indices of matrix, vector, and scalar type for the static diact effects, utilities, and residence times are introduced systematically as quantitative ecosystem indicators further below in this section. A quantitative technique for the analysis of food chains and webs, as well as the characterization and classification of interspecific interactions and the determination of their strength is then developed based on the diact effect and utility measures at the end of this section. 
2.1. System partitioning methodology. The standard governing equations for compartmental dynamics are

$$
\dot{x}_{i}(t)=\left(z_{i}(t, x)+\sum_{j=1}^{n} f_{i j}(t, x)\right)-\left(y_{i}(t, x)+\sum_{j=1}^{n} f_{j i}(t, x)\right)
$$

with the initial conditions $x_{i}\left(t_{0}\right)=x_{i, 0}$, for $i=1, \ldots, n$. The concepts and notations employed in this formulation are as follows:

$\begin{array}{ll}n & \text { number of compartments } \\ t & \text { time }[\mathrm{t}] \\ x_{i}(t) & \text { total material (mass) [m] (or energy, currency) in compart- } \\ & \text { ment } i, i=1, \ldots, n, \text { at time } t \\ f_{i j}(t, x) & \text { non-negative flow from compartment } j \text { to } i, \text { at time } t[\mathrm{~m} / \mathrm{t}] \\ y_{i}(t, x)=f_{0 i}(t, x) & \text { environmental output from compartment } i \text { at time } t \\ z_{i}(t, x)=f_{i 0}(t, x) & \text { environmental }(j=0) \text { input into compartment } i \text { at time } t\end{array}$

For notational convenience, we define a direct flow matrix function $F$ of size $n \times n$ as

$$
F(t, x)=\left(f_{i j}(t, x)\right)
$$

and the inward and outward throughflow vector functions as

$$
\begin{aligned}
& \check{\tau}(t, x)=\left[\check{\tau}_{1}(t, x), \ldots, \check{\tau}_{n}(t, x)\right]^{T}=z(t, x)+F(t, x) \mathbf{1} \text { and } \\
& \hat{\tau}(t, x)=\left[\hat{\tau}_{1}(t, x), \ldots, \hat{\tau}_{n}(t, x)\right]^{T}=y(t, x)+F^{T}(t, x) \mathbf{1},
\end{aligned}
$$

respectively, where

$$
z(t, x)=\left[z_{1}(t, x), \ldots, z_{n}(t, x)\right]^{T} \quad \text { and } \quad y(t, x)=\left[y_{1}(t, x), \ldots, y_{n}(t, x)\right]^{T}
$$

are the input and output vector functions, $\mathbf{1}$ denotes the column vector of size $n$ whose entries are all one, and the superscript $T$ represents the matrix transpose..

The system partitioning methodology yields the governing equations for subcompartmental dynamics as follows (see Figs. 1 and 2):

$$
\dot{x}_{i_{k}}(t)=\left(z_{i_{k}}(t, \mathrm{x})+\sum_{j=1}^{n} f_{i_{k} j_{k}}(t, \mathrm{x})\right)-\left(y_{i_{k}}(t, \mathrm{x})+\sum_{j=1}^{n} f_{j_{k} i_{k}}(t, \mathrm{x})\right)
$$

for $i=1, \ldots, n, k=0, \ldots, n$, with the initial conditions

$$
x_{i_{k}}\left(t_{0}\right)=\left\{\begin{array}{rr}
x_{i, 0}, & k=0 \\
0, & k \neq 0
\end{array}\right.
$$

where

$$
\mathrm{x}(t)=\left[x_{1_{0}}(t), \ldots, x_{n_{0}}(t), x_{1_{1}}(t), \ldots, x_{n_{1}}(t), \ldots, x_{1_{n}}(t), \ldots, x_{n_{n}}(t)\right]^{T} .
$$

The relationship between the compartmental and subcompartmental flows and storages can be stated as

$$
x_{i}(t)=\sum_{k=0}^{n} x_{i_{k}}(t) \quad \text { and } \quad f_{i j}(t, x)=\sum_{k=0}^{n} f_{i_{k} j_{k}}(t, \mathrm{x})
$$



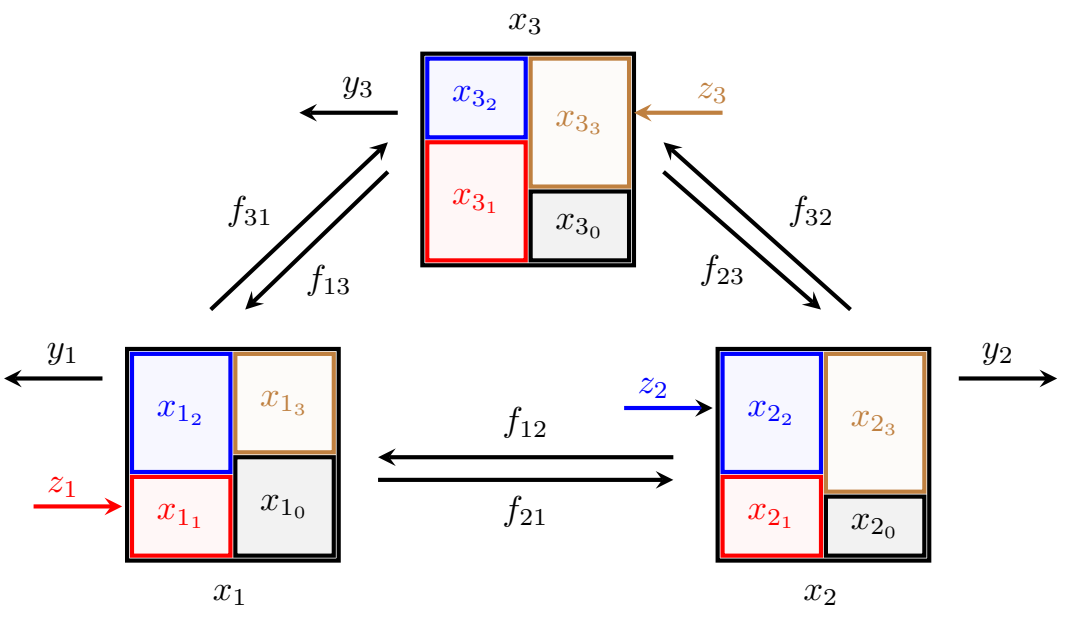

FIG. 1. Schematic representation of the input-oriented dynamic subcompartmentalization in a three-compartment model system. Each subsystem is colored differently; the second subsystem $(k=2)$ is blue, for example. Only the subcompartments in the same subsystem $\left(x_{1_{2}}(t), x_{2_{2}}(t)\right.$, and $x_{3_{2}}(t)$ in the second subsystem, for example) interact with each other. Subsystem $k$ receives environmental input only at subcompartment $k_{k}$. The initial subsystem receives no environmental input. The dynamic flow partitioning is not represented in this figure. Compare this figure with Fig. 2, in which the subcompartmentalization and the corresponding flow partitioning are illustrated for $x_{1}(t)$ only.

where

$$
f_{i_{k} j_{k}}(t, \mathrm{x})=x_{j_{k}}(t) \frac{f_{i j}(t, x)}{x_{j}(t)}=d_{j_{k}}(\mathrm{x}) f_{i j}(t, x)
$$

and the decomposition factor is defined as $d_{j_{k}}(\mathrm{x})=x_{j_{k}}(t) / x_{j}(t)$.

The concepts and notations used in the system partitioning methodology are summarized below:

$$
\begin{array}{ll}
x_{i_{k}}(t) & \text { storage generated by environmental input } z_{k}(t, \mathrm{x}) \text { during } \\
& {\left[t_{0}, t\right] \text { and stored in subcompartment } k \text { of compartment } i,} \\
& \text { that is, in subcompartment } i_{k}, k=0, \ldots, n, \text { at time } t \\
& \text { non-negative flow from subcompartment } j_{k} \text { to } i_{k} \text { at time } t \\
f_{i_{k} j_{k}}(t, \mathrm{x}) & \text { environmental }(j=0) \text { output from subcompartment } i_{k} \text { at } \\
y_{i_{k}}(t, \mathrm{x})=f_{0 i_{k}}(t, \mathrm{x}) \quad \text { time } t & \text { environmental input into subcompartment } i_{k} \text { at time } t, \\
z_{i_{k}}(t, \mathrm{x})=\delta_{i k} z_{i}(t, x) & \text { where } \delta_{i k} \text { is the discrete delta function }
\end{array}
$$

If the original system, Eq. 2.1, is linear, the decomposed system, Eq. 2.3, can be expressed in the following linear matrix form:

$$
\begin{array}{ll}
\dot{X}(t)=\check{T}(t)-\hat{T}(t), & X\left(t_{0}\right)=\mathbf{0}, \\
\dot{x}_{0}(t)=\check{\tau}_{0}(t)-\hat{\tau}_{0}(t), & x_{0}\left(t_{0}\right)=x_{0},
\end{array}
$$

where

$$
X_{i k}(t)=x_{i_{k}}(t), \quad \check{T}_{i k}(t)=\check{\tau}_{i_{k}}(t), \quad \text { and } \quad \hat{T}_{i k}(t)=\hat{\tau}_{i_{k}}(t)
$$




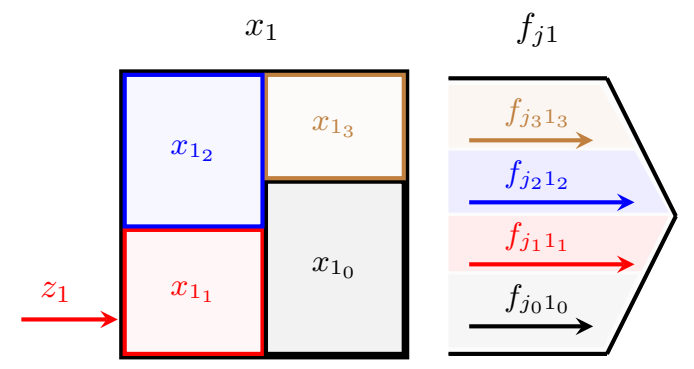

FIG. 2. Schematic representation of the dynamic flow partitioning in a three-compartment model system. The figure illustrates subcompartmentalization of compartment $i=1$ and the corresponding dynamic flow partitioning from this compartment to others, $j$.

for $i, k=1, \ldots, n$; the inward and outward subthroughflows are

$$
\check{\tau}_{i_{k}}(t)=z_{i_{k}}(t)+\sum_{j=1}^{n} f_{i_{k} j_{k}}(t) \quad \text { and } \quad \hat{\tau}_{i_{k}}(t)=y_{i_{k}}(t)+\sum_{j=1}^{n} f_{j_{k} i_{k}}(t),
$$

for $k=0,1, \ldots, n$; and the storage, inward, and outward throughflow vector functions for the initial subsystem are $x_{0}(t)=\left[x_{1_{0}}(t), \ldots, x_{n_{0}}(t)\right]^{T}, \check{\tau}_{0}(t)=\left[\check{\tau}_{1_{0}}(t), \ldots, \check{\tau}_{n_{0}}(t)\right]^{T}$, and $\hat{\tau}_{0}(t)=\left[\hat{\tau}_{1_{0}}(t), \ldots, \hat{\tau}_{n_{0}}(t)\right]^{T}$, respectively. We use the constant notation $x_{0}$ for the constant initial conditions and the function notation $x_{0}(t)$ for the evolution of these initial conditions with $x_{0}\left(t_{0}\right)=x_{0}$.

The governing system, Eq. 2.6, can alternatively be expressed as follows:

$$
\begin{aligned}
\dot{X}(t) & =\mathcal{Z}(t)+A(t) X(t), & & X\left(t_{0}\right)=\mathbf{0} \\
\dot{x}_{0}(t) & =A(t) x_{0}(t), & & x_{0}\left(t_{0}\right)=x_{0}
\end{aligned}
$$

where

$$
A(t)=\left(F(t)-\mathcal{Y}(t)-\operatorname{diag}\left(F^{T}(t) \mathbf{1}\right)\right) \mathcal{X}^{-1}(t),
$$

and the diagonal storage, input, and output matrices are

$$
\mathcal{X}(t)=\operatorname{diag}(x(t)), \quad \mathcal{Y}(t)=\operatorname{diag}(y(t)), \quad \text { and } \quad \mathcal{Z}(t)=\operatorname{diag}(z(t)) .
$$

The notation $\operatorname{diag}(x(t))$ represents the diagonal matrix whose diagonal elements are the elements of vector $x(t)$, and $\operatorname{diag}(X(t))$ represents the diagonal matrix whose diagonal elements are the same as the diagonal elements of matrix $X(t)$.

2.2. Static ecological system analysis. The static version of the dynamic methodology summarized above has recently been introduced by [14]. This static methodology will be summarized in this section.

At steady state, the time derivatives are zero and all system flows and storages are constant. That is,

$$
\dot{X}(t)=\mathbf{0} \quad \text { and } \quad \dot{x}_{0}(t)=\mathbf{0} .
$$

We will use the same notation, $\mathbf{0}$, for both zero matrix and zero vector for notational simplicity. If the partitioned system is at steady state, the original system is also at steady state. The constant static quantities will be denoted by the same symbols without the time argument, that is $X(t)=X$, for example. 
For linear systems, $A$ is a strictly diagonally dominant constant matrix and, therefore, is invertible. It can be expressed as

$$
A=(F-\mathcal{T}) \mathcal{X}^{-1}=F \mathcal{X}^{-1}-\mathcal{T} \mathcal{X}^{-1}=Q^{x}-\mathcal{R}^{-1}
$$

where $Q^{x}=F \mathcal{X}^{-1}, \mathcal{R}=\mathcal{X} \mathcal{T}^{-1}$, and $\mathcal{T}=\operatorname{diag}(\hat{\tau})=\mathcal{Y}+\operatorname{diag}\left(F^{T} \mathbf{1}\right)$. The $n \times$ $n$ matrix $A$ will be called the flow intensity matrix per unit storage. The novel matrix measure $\mathcal{R}$ will be called the residence time matrix and discussed in detail in Section 2.8.

We then have the following solutions to Eq. 2.9 for the substorage matrix, $X$, and initial substorage vector, $x_{0}$, at steady state:

$$
X=-A^{-1} \mathcal{Z}=\mathcal{X}(\mathcal{T}-F)^{-1} \mathcal{Z} \quad \text { and } \quad x_{0}=\mathbf{0} .
$$

Since the inward and outward throughflows at each subcompartment are the same at steady state, we have

$$
\tau=\check{\tau}=\hat{\tau} \quad \text { and } \quad T=\check{T}=\hat{T} .
$$

The static subthroughflow matrices, $\check{T}$ and $\hat{T}$, and the initial subthroughflow vector, $\tau_{0}$, can then be expressed as

$$
\begin{aligned}
T=\check{T} & =\mathcal{Z}+F \mathcal{X}^{-1} X, \\
\hat{T} & =\left(\mathcal{Y}+\operatorname{diag}\left(F^{T} \mathbf{1}\right)\right) \mathcal{X}^{-1} X=\mathcal{T} \mathcal{X}^{-1} X=\mathcal{R}^{-1} X, \\
\tau_{0} & =\check{\tau}_{0}=\hat{\tau}_{0}=\mathbf{0} .
\end{aligned}
$$

Therefore, the static system partitioning methodology yields the subthroughflow and substorage matrices, $T$ and $X$, formulated in Eqs. 2.12 and 2.14. They quantify the distribution of environmental inputs and the organization of the associated storages generated by the inputs within the system. More specifically, the $(i, k)$-elements of these matrices, $\tau_{i_{k}}$ and $x_{i_{k}}$, are the throughflow at and storage in compartment $i$, generated by the environmental input into compartment $k, z_{k}$.

In order to analyze the system behavior per unit input at steady state, all but the initial subsystems are scaled by their corresponding positive environmental input, $z_{k}>0$ ( $\mathcal{Z}$ is invertible). The scaled substorage and subthroughflow matrices, $S$ and $N$, will be defined as follows:

$$
S=X \mathcal{Z}^{-1} \quad \text { and } \quad N=T \mathcal{Z}^{-1}
$$

Componentwise, they can be expressed as $S_{i k}=s_{i_{k}}=x_{i_{k}} / z_{k}$ and $N_{i k}=n_{i_{k}}=$ $\tau_{i_{k}} / z_{k}$. Note that the physical dimension of $S$ is time [t], and $N$ is dimensionless. As immediate consequences of these definitions, we have

$$
X=S \mathcal{Z} \Rightarrow x=S z \text { and } T=N \mathcal{Z} \Rightarrow \tau=N z
$$

These relationships refine and extend the static network analysis from the current compartmental level to the subcompartmental level. Both input- and output-oriented subsystem scaling are formulated by [14].

The invertible matrix measures, $S$ and $N$, will be called the cumulative strorage and flow distribution matrices, respectively. They can be expressed in the following various forms:

$$
\begin{aligned}
& N=I+Q^{x} S=\mathcal{R}^{-1} S=\left(I-Q^{\tau}\right)^{-1} \\
& S=-A^{-1}=\left(\mathcal{R}^{-1}-Q^{x}\right)^{-1}=\mathcal{R}\left(I-Q^{\tau}\right)^{-1}=(I-L)^{-1} \mathcal{R}
\end{aligned}
$$


TABLE 1

The input-oriented, flow-based diact flow and storage distribution and the simple and composite diact (sub)flow and (sub)storage matrices. The superscript $\left(^{*}\right)$ in each equation represents any of the diact symbols.

\begin{tabular}{|c|c|c|c|c|}
\hline diact & flow and storage distributio & matrices & flows & storages \\
\hline $\mathrm{d}$ & $N^{\mathrm{d}}=F \mathcal{T}^{-1}$ & \multirow{5}{*}{$S^{*}=\mathcal{R} N^{*}$} & \multirow{5}{*}{$\begin{array}{l}T^{*}=N^{*} \mathcal{T} \\
T_{\ell}^{*}=N^{*} \mathcal{T}_{\ell} \\
\tilde{T}^{*}=N^{*} \mathrm{~T}\end{array}$} & \multirow{5}{*}{$\begin{aligned} X^{*} & =S^{*} \mathcal{T} \\
X_{\ell}^{*} & =S^{*} \mathcal{T} \\
\tilde{X}^{*} & =S^{*} \mathrm{~T}\end{aligned}$} \\
\hline$i$ & $N^{\mathrm{i}}=(N-I) \mathcal{N}^{-1}-F \mathcal{T}^{-1}$ & & & \\
\hline a & $N^{\mathrm{a}}=\left(\mathcal{N}^{-1} N-I\right) \mathcal{N}^{-1}$ & & & \\
\hline c & $N^{c}=\left(N-\mathcal{N}^{-1} N\right) \mathcal{N}^{-1}$ & & & \\
\hline$t$ & $N^{\mathrm{t}}=(N-I) \mathcal{N}^{-1}$ & & & \\
\hline
\end{tabular}

where the flow and storage distribution matrices $Q^{\tau}$ and $L$ are

$$
Q^{\tau}=F \mathcal{T}^{-1}=Q^{x} \mathcal{R} \text { and } L=\mathcal{R} Q^{x} .
$$

The equivalence of the proposed formulations for $S$ and $N$ to the ones in the current static ecological network analyses is shown by [14].

The subthroughflow and substorage matrices can be expressed as infinite geometric series of the distribution matrices. That is,

$$
T=N \mathcal{Z}=\left(I-Q^{\tau}\right)^{-1} \mathcal{Z}=\left(\sum_{m=0}^{\infty}\left(Q^{\tau}\right)^{m}\right) \mathcal{Z},
$$

where $\left(Q^{\tau}\right)^{0}=I$, and

$$
X=S \mathcal{Z}=\mathcal{R}\left(I-Q^{\tau}\right)^{-1} \mathcal{Z}=(I-L)^{-1} \mathcal{R} \mathcal{Z}=\left(\sum_{m=0}^{\infty} L^{m}\right) \mathcal{R} \mathcal{Z} .
$$

The geometric series expansion of $S$ and $N$, as formulated in Eqs. 2.17 and 2.18, were also proposed in the literature with different derivations and interpretations $[23,19,17]$. Instead of the diagonal matrix $\mathcal{R}$ in the proposed formulation for $S$, however, the authors used a scalar time interval $\Delta t \in[0,1]$ through dimension analysis, which cannot describe system dynamics accurately. Moreover, the indirect flow contributions were defined as the flow contributions carried by subsequent steps after their first entrance into a compartment, as discussed in Section 2.6. Therefore, the higher-powered terms $(m>1)$ in the series expansions are considered as indirect contributions in these formulations [39, 41, 23, 54, 4, 33]. Even the direct transactions between compartments after the first step $(m=1)$ are counted as indirect. Therefore, these microscopic formulations cannot accurately describe the indirect transactions as demonstrated theoretically and through a case study by [14].

The system partitioning methodology introduced in this section is based on environmental inputs. The output-oriented system analysis can be formulated in parallel [14]. The output-oriented quantities are represented by "barred" notations of their input-oriented counterparts. For example, the output-oriented cumulative flow and storage distribution matrices are denoted by $\bar{N}$ and $\bar{S}$, respectively. The duality of input- and output-oriented analyses through novel similarity relationships between the corresponding pairs of matrix measures can be expressed as follows:

$$
S \mathcal{X}=\mathcal{X} \bar{S}^{T} \quad \text { and } \quad N \mathcal{T}=\mathcal{T} \bar{N}^{T},
$$

using Eq. 2.16 and its output-oriented counterpart formulated by [14]. The relationships between the pairs of $(X, \bar{X})$ and $(T, \bar{T})$ are also formulated in Eq. 2.28. 


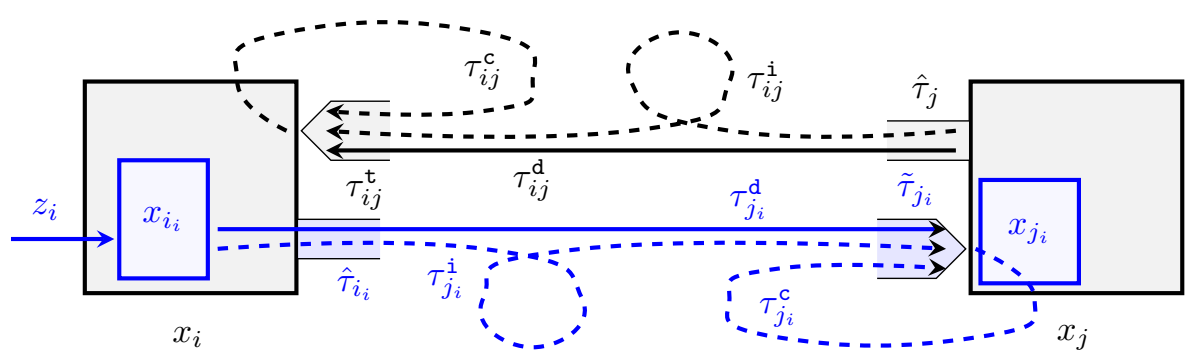

FIG. 3. Schematic representation of the composite and simple diact flows. Solid arrows represent direct flows, and dashed arrows represent indirect flows through other compartments (not shown). The composite diact flows (black) generated by outward throughflow $\hat{\tau}_{j}$ (i.e. derived from all environmental inputs): direct flow, $\tau_{i j}^{d}=f_{i j}$, indirect flow, $\tau_{i j}^{i}$, acyclic flow, $\tau_{i j}^{a}=\tau_{i j}^{t}-\tau_{i j}^{c}$, cycling flow, $\tau_{i j}^{c}$, and transfer flow, $\tau_{i j}^{t}$. The simple diact flows (blue) generated by outward subthroughflow $\hat{\tau}_{i_{i}}$ (i.e. derived from single environmental input $z_{i}$ ): direct flow, $\tau_{j_{i}}^{d}=\tau_{j_{i} i_{i}}^{d}=f_{j_{i} i_{i}}$, indirect flow, $\tau_{j_{i}}^{i}=\tau_{j_{i} i_{i}}^{i}$, acyclic flow, $\tau_{j_{i}}^{a}=\tau_{j_{i} i_{i}}^{a}=\tilde{\tau}_{j_{i}}-\tau_{j_{i}}^{c}$, cycling flow, $\tau_{j_{i}}^{c}=\tau_{j_{i} i_{i}}^{c}$, and transfer flow, $\tau_{j_{i}}^{t}=\tilde{\tau}_{j_{i}}=\check{\tau}_{j_{i}}-z_{j_{i}}$. Note that the cycling flows at the terminal (sub)compartment may include the segments of the direct and/or indirect flows at that (sub)compartment, if the cycling flows indirectly pass through the corresponding initial (sub)compartment. Threfore, the acyclic flows are composed of the segments of the direct and/or indirect flows.

2.3. The diact flows and storages. For intercompartmental flow and storage dynamics, the dynamic direct, indirect, acyclic, cycling, transfer (diact) flows and storages, as well as their static versions have recently been introduced through the subsystem partitioning methodology by $[11,14]$.

The composite transfer flow will be defined as the total intercompartmental transient flow from one compartment, directly or indirectly through other compartments, to another. The composite direct, indirect, acyclic and cycling flows from the initial compartment to the terminal compartment are then defined as the direct, indirect, non-cycling, and cycling segments at the terminal compartment of the transfer flow (see Fig. 3). These composite diact flows at the terminal compartment are generated by the outward throughflow at the initial compartment derived from all environmental inputs. The diact flow generated at the terminal subcompartment by the outward subthroughflow at the input-receiving subcompartment of the initial compartment derived from the corresponding single environemtnal input will be called the simple diact flow. Therefore, the simple transfer flow is the intercompartmental subthroughflow at the terminal subcompartment. The simple direct, indirect, acyclic, and cycling flows from the initial subcompartment to the terminal subcompartment are then defined as the direct, indirect, non-cycling, and cycling segments of the intercompartmental subthroughflow at the terminal subcompartment (see Fig. 3). The associated simple and composite diact storages are then defined as the storages generated by the corresponding diact flows.

The input-oriented, flow-based, simple and composite diact flow and storage distribution matrices and the corresponding flow and storage matrices are listed in Table 1 at both subcompartmental and compartmental levels. Each transaction type is denoted by the corresponding diact superscript. In a nutshell, by left multiplying the (sub)throughflow matrices, the diact distribution matrices yield the corresponding diact (sub)flow and (sub)storage matrices. The output-oriented, flow-based, simple and composite diact flow and storage distribution matrices and the corresponding flow and storage matrices are also listed in Table 2. The input- and output-oriented 
TABLE 2

The output-oriented, flow-based diact flow and storage distribution and the simple and composite diact (sub)flow and (sub)storage matrices. The superscript $\left(^{*}\right)$ in each equation represents any of the diact symbols.

\begin{tabular}{|c|c|c|c|c|}
\hline diact & flow and storage distribution & matrices & flows & storages \\
\hline d & $\bar{N}^{\mathrm{d}}=F^{T} \mathcal{T}^{-1}$ & & & \\
\hline$i$ & $\bar{N}^{i}=(\bar{N}-I) \mathcal{N}^{-1}-F^{T} \mathcal{T}^{-1}$ & & $\bar{T}^{*}=\bar{N}^{*} \mathcal{T}$ & $\bar{X}^{*}=\bar{S}^{*} \mathcal{T}$ \\
\hline a & $\bar{N}^{\mathrm{a}}=\left(\mathcal{N}^{-1} \overline{\bar{N}}-I\right) \mathcal{N}^{-1}$ & $\bar{S}^{*}=\mathcal{R} \bar{N}^{*}$ & $\bar{T}_{\ell}^{*}=\bar{N}^{*} \overline{\mathcal{T}}_{\ell}$ & $\bar{X}_{\tilde{\nu}}^{*}=\bar{S}^{*} \overline{\mathcal{T}}_{\ell}$ \\
\hline c & $\bar{N}^{c}=\left(\bar{N}-\mathcal{N}^{-1} \bar{N}\right) \mathcal{N}^{-1}$ & & $\bar{T}^{*}=\bar{N}^{*} \bar{\top}$ & $\bar{X}^{*}=\bar{S}^{*} \overline{\mathrm{T}}$ \\
\hline $\mathrm{t}$ & $\bar{N}^{\mathrm{t}}=(\bar{N}-I) \mathcal{N}^{-1}$ & & & \\
\hline
\end{tabular}

storage-based diact flow and storage matrices are formulated by [14].

2.4. Holistic view of ecosystems. The mathematical theory recently introduced for dynamic analysis of nonlinear compartmental systems constructs a base for the development of new system analysis tools [13]. Based on this theory, multiple system measures and indices have recently been developed as quantitative ecological indicators $[11,12]$. The static version of the proposed dynamic methodology has also been recently introduced by [14].

In the present manuscript, multiple measures and indices of matrix, vector, and scalar type for the static diact effects, utilities, and residence times are introduced integrally and systematically. Current major system measures and indices of static ecological network analysis are also reformulated with a different derivation rationale in the context of the proposed methodology and, therefore, are integrated with all the other novel system analysis tools introduced in the present work. Corrections in some current formulations are also suggested, as discussed in Sections 2.2 and 2.6.

In addition to the novel system measures and indices, unifying relationships between some current static measures are also unveiled through the proposed methodology. The cumulative storage and throughflow distribution matrices, $S$ and $N$, have been treated separately in the literature. It is shown, however, in Eq. 2.20 that they are related by a factor of the residence time matrix, $\mathcal{R}$ - a novel system measure introduced in Section 2.8. The residence time matrix also combines and integrates the input- and output- oriented compartmental and subcompartmental matrix and vector measures as outlined below in Eqs. 2.21 and 2.25. Therefore, $\mathcal{R}$ has a central role in connecting various measures and integrating different components of the proposed methodology.

The duality of input- and output-oriented system analyses established through similarity relationships between the cumulative storage and throughflow distribution matrices, as formulated in Eq. 2.19, integrates the system analyses in both orientations. The system and subsystem partitioning methodologies are also integrated through the geometric series expansions of the distribution matrices, as demonstrated in Eqs. 2.17 and 2.18. A number of equivalent formulations for $S$ and $N$ are also presented in Eq. 2.16, which combines multiple matrix measures all together. Consequently, various components of ecosystem analysis are effectively combined and holistically integrated within the proposed unifying mathematical framework. As a result, the proposed methodology is the first comprehensive approach in literature that leads to a holistic methodology for ecosystem analysis. The details of this holistic perspective of the static theory are presented in this section.

The matrix measures, $S$ and $N$, are treated separately in the environ theory [19]. 
The proposed methodology, however, unveils a unique relationship that combines these two matrix measures effectively. Based on the subsystem scaling argument presented above in Section 2.2, Eq. 2.14 implies that

$$
T=\mathcal{R}^{-1} X \Rightarrow S=\mathcal{R} N \text {. }
$$

Therefore, these matrix measures are related in a unique and natural way, since the storage distribution, $S$, generated by the throughflow distribution per unit input, $N$, is controlled by the residence time factor, $\mathcal{R}$.

The system components, such as the compartmental vector and subcompartmental matrix measures can also be combined and integrated within the proposed unifying framework by the residence time matrix:

$$
x=S z=\mathcal{R} N z=\mathcal{R} \tau \quad \text { and } \quad X=S \mathcal{Z}=\mathcal{R} N \mathcal{Z}=\mathcal{R} T .
$$

These relationships provide a compact summary of static ecological network theory. They indicate that $\mathcal{R}$ can be interpreted as a linear transformation that maps throughflows, $\tau$, to storages, $x$, or subthroughflows, $T$, to substorages, $X$.

The residence time matrix can be expressed in various forms using Eqs. 2.14, 2.16, and 2.20 as follows:

$$
\mathcal{X} \mathcal{T}^{-1}=X T^{-1}=\mathcal{R}=S N^{-1}=\left(S^{-1}+Q^{x}\right)^{-1} .
$$

Note that the inverse of $n \times n$ diagonal residence time matrix, $\mathcal{R}$, is the outward throughflow intensity matrix, $\mathcal{R}^{-1}=\mathcal{T} \mathcal{X}^{-1}$. Using the diact distribution matrices presented in Section 2.3, the residence time matrix can alternatively be expressed further as

$$
\mathcal{R}=\mathcal{X}_{k} \mathcal{T}_{k}^{-1}=S^{\mathrm{t}}\left(N^{\mathrm{t}}\right)^{-1}=\left(Q^{x}\right)^{-1} Q^{\tau}
$$

where the diagonal $k^{\text {th }}$ storage and subthroughflow matrices are

$$
\mathcal{X}_{k}=\operatorname{diag}\left(\left[x_{1_{k}}, \ldots, x_{n_{k}}\right]\right) \quad \text { and } \quad \check{\mathcal{T}}_{k}=\operatorname{diag}\left(\left[\check{\tau}_{1_{k}}, \ldots, \check{\tau}_{n_{k}}\right]\right),
$$

respectively, as formulated by [14], and the invertibility of $\mathcal{T}_{k}, N^{\mathrm{t}}$, and $Q^{x}$ is assumed. The other diact distribution matrices can be used instead of the transfer distribution matrices, $S^{\mathrm{t}}$ and $N^{\mathrm{t}}$, in Eq. 2.23.

This holistic view of ecological networks can further be extended to outputoriented analysis. As given for the input-oriented measures in Eq. 2.21, the system components, such as the output-oriented compartmental vector and subcompartmental matrix measures, can also be integrated by the residence time matrix, $\mathcal{R}=\overline{\mathcal{R}}$, as follows:

$$
x=\bar{S} y=\mathcal{R} \bar{N} y=\mathcal{R} \tau \quad \text { and } \quad \bar{X}=\bar{S} \mathcal{Y}=\mathcal{R} \bar{N} \mathcal{Y}=\mathcal{R} \bar{T} .
$$

These relationships provide a compact summary of output-oriented static ecological system theory. The residence time matrix can be expressed in various additional forms using the output-oriented measures similar to their input-oriented counterparts given in Eq. 2.22 as follows:

$$
\mathcal{X} \mathcal{T}^{-1}=\bar{X} \bar{T}^{-1}=\mathcal{R}=\bar{S} \bar{N}^{-1}=\left(\bar{S}^{-1}+\bar{Q}^{x}\right)^{-1} .
$$

Alternatively, $\mathcal{R}$ can also be written as

$$
\mathcal{R}=\mathcal{X}_{k} \mathcal{T}_{k}^{-1}=\bar{S}^{\mathrm{t}}\left(\bar{N}^{\mathrm{t}}\right)^{-1}=\left(\bar{Q}^{x}\right)^{-1} \bar{Q}^{\tau}
$$


where $\bar{Q}^{\tau}=F^{T} \mathcal{T}^{-1}$, and the invertibility of $\overline{\mathcal{T}}_{k}=\mathcal{T}_{k}, \bar{N}^{\mathrm{t}}$, and $\bar{Q}^{x}=F^{T} \mathcal{X}^{-1}$ is assumed. As seen from the relationships presented above, the residence time matrix, $\mathcal{R}$, does not only connect the vector and matrix measures in Eqs. 2.21 and 2.25, it also combines the input- and output-oriented measures as shown in Eqs. 2.22 and 2.26 and compartmental and subcompartmental measures as given in Eqs. 2.23 and 2.27. Consequently, $\mathcal{R}$ has a critical role in the integration of static system measures within the proposed framework and, therefore, in the holistic view of ecosystems.

The similarity of the input- and output-oriented cumulative storage and flow distribution matrices is formulated in Eq. 2.19. This duality establishes additional connections between the input- and output-oriented matrix measures. As examples, the relationships between input- and output-oriented substorage and subthroughflow matrices can be expressed as

$$
X=\left(\mathcal{X} \mathcal{Y}^{-1}\right) \bar{X}^{T}\left(\mathcal{Z} \mathcal{X}^{-1}\right) \quad \text { and } \quad T=\left(\mathcal{T} \mathcal{Y}^{-1}\right) \bar{T}^{T}\left(\mathcal{Z} \mathcal{T}^{-1}\right),
$$

using Eqs. 2.19 and 2.20.

In summary, the relationships introduced in this section effectively combine various components of the proposed methodology altogether and holistically integrate major static measures of both input- and output-oriented approaches within the proposed unifying framework, which leads to a holistic mathematical methodology for static ecological network analysis.

The next section briefly discusses the system measures developed in the previous sections, such as the substorage and subthroughflow matrices, as well as the diact flows and storages. Multiple new static measures and indices for the direct, indirect, acyclic, cycling, and transfer (diact) effects, utilities, and residence times are formulated systematically in the following sections. Ecological implications of the proposed measures and indices are also discussed in the corresponding sections. The efficiency and wide applicability of these system analysis tools are then presented through the case studies in Section 3.

2.5. System Measures. The proposed mathematical method is based on the novel analytical and explicit, mutually exclusive and exhaustive system and subsystem partitioning methodologies $[13,11,14]$. While the system partitioning yields the subthroughflow and substorage matrices for the determination of environmental influence on system compartments in terms of the flow and storage generation, the subsystem partitioning yields the transient and diact flow and storage matrices for the quantification of intercompartmental flow and storage dynamics.

The elements of the subthroughflow and substorage matrices, $T$ and $X$, represent the distribution of environmental inputs and the organization of the associated storages generated by the inputs within the system. More precisely, $\tau_{i_{k}}$ and $x_{i_{k}}$ represent the subthroughflow and substorage in compartment $i$ generated by the environmental input into compartment $k, z_{k}$ (see Fig. 2). In other words, the proposed methodology can partition composite compartmental throughflows and associated storages into subcompartmental segments based on their constituent environmental inputs of the same conserved quantity. This partitioning enables tracking the fate of environmental inputs and the associated storages generated by the inputs individually and separately within the system. The discussion above is valid also for the outputoriented subthroughflow and substorage matrices, $\bar{T}$ and $\bar{X}$. Note that the composite compartmental net throughflows and storages, $\tau_{i}$ and $x_{i}$, cannot be used to distinguish the portions of these throughflows and storages derived from individual environmental inputs separately. Therefore, the solution to the decomposed system brings out 
inferences that cannot be obtained through the analysis of the original system by the state-of-the-art techniques.

The transient flows and associated storages transmitted along a given flow path are also formulated through the subsystem partitioning methodology. Therefore, the subsystem partitioning determines the distribution of arbitrary intercompartmental flows and the organization of the associated storages generated by these flows along given subflow paths within the subsystems. Consequently, arbitrary composite intercompartmental flows and storages can be decomposed into the constituent transient subflow and substorage segments along a given set of subflow paths. In other words, the subsystem decomposition enables tracking the fate of arbitrary intercompartmental flows and associated storages within the subsystems. Based on the concept of transient flow and storage, the direct, indirect, acyclic, cycling, transfer (diact) flows and storages transmitted from one compartment, directly or indirectly, to any other -including itself - within the system are also systematically formulated.

In what follows, multiple new static measures and indices for the diact effects, utilities, and residence times are formulated systematically.

2.6. The diact effect measures and indices. The effect of one compartment on another through direct transactions is relatively easier to analyze. The proposed subsystem partitioning methodology also enables the determination of the indirect effect of one subcompartment indirectly through other subcompartments on another or itself within a complex network. In fact, parallel to the definitions of diact flows and storages, we systematically introduce all the other diact effect measures and indices in this section. These system analysis tools are the static version of the dynamic diact effect measures and indices recently proposed by [12].

Based on the transfer flows introduced in Section 2.3, the transfer effect measures and indices of matrix, vector, and scalar types are formulated below at the compartmental level. The subcompartmental level formulations in parallel are straightforward using the transfer subflows and substorages instead of the transfer flows and storages.

The flow-based transfer effect index is defined as the transfer flow normalized by total system throughflow. The storage-based transfer effect index is defined similarly, using the transfer storage instead of the flow and total system storage for normalization. Therefore, the flow- and storage-based transfer effect indices are fractions of total system throughflow and storage, respectively. Both flow- and storage-based effect indices quantify the influence of system compartments on each other.

The flow- and storage-based composite transfer effect indices of a set of compartments, $K$, on another set, $I, \mathrm{t}_{I K}^{\tau}$ and $\mathrm{t}_{I K}^{x}$, can be formulated as the fraction of total system throughflow that is initiated at compartments $K$ and transmited directly or indirectly to $I$, and as the fraction of total system storage generated by these transfer flows, respectively. That is,

$$
\mathrm{t}_{I K}^{\tau}=\frac{\sum_{i \in I} \sum_{k \in K} \tau_{i k}^{\mathrm{t}}}{\sum_{i=1}^{n} \check{\tau}_{i}} \quad \text { and } \quad \mathrm{t}_{I K}^{x}=\frac{\sum_{i \in I} \sum_{k \in K} x_{i k}^{\mathrm{t}}}{\sum_{i=1}^{n} x_{i}}
$$

where $I, K \subseteq\{1, \ldots, n\}$. If the sets $I$ and $K$ have one element, that is, $I=\{i\}$ and $K=\{k\}$, transfer effect indices will be denoted by $\mathrm{t}_{i k}^{\tau}$ and $\mathrm{t}_{i k}^{x}$. Note that the transfer effect indices are dimensionless.

The flow- and storage-based composite transfer effect matrices will be denoted by $\mathrm{T}^{\tau}=\left(\mathrm{t}_{i k}^{\tau}\right)$ and $\mathrm{T}^{x}=\left(\mathrm{t}_{i k}^{x}\right)$ and formulated in matrix form as

$$
\mathrm{T}^{\tau}=\frac{1}{\sigma^{\tau}} T^{\mathrm{t}} \quad \text { and } \quad \mathrm{T}^{x}=\frac{1}{\sigma^{x}} X^{\mathrm{t}}
$$


where the scalar system indices $\sigma^{\tau}=\mathbf{1}^{T} \check{\tau}=\mathbf{1}^{T} \hat{\tau}$ and $\sigma^{x}=\mathbf{1}^{T} x$ are called the total system throughflow and storage, respectively. It is worth noting that the input-oriented total system throughflow and storage are equal to their output-oriented counterparts [14]. The flow-based composite transfer effect of the system on the compartments, $\check{t}^{\tau}$, and those of the compartments on the system, $\hat{t}^{\tau}$, will be defined as vector measures:

$$
\check{\mathrm{t}}^{\tau}=\mathrm{T}^{\tau} \mathbf{1} \text { and } \hat{\mathrm{t}}^{\tau}=\mathbf{1}^{T} \mathrm{~T}^{\tau} \text {. }
$$

The storage-based composite transfer effect vectors can be defined similarly. We will use the notations $t^{\tau}$ and $t^{x}$ for the sum of the transfer effects of the entire system on all compartments, that is for $I=K=\{1, \ldots, n\}$. They can be formulated as follows:

$$
\mathrm{t}^{\tau}=\frac{\mathbf{1}^{T} T^{\mathrm{t}} \mathbf{1}}{\sigma^{\tau}}=\mathbf{1}^{T} \mathrm{~T}^{\tau} \mathbf{1} \text { and } \mathrm{t}^{x}=\frac{\mathbf{1}^{T} X^{\mathrm{t}} \mathbf{1}}{\sigma^{x}}=\mathbf{1}^{T} \mathrm{~T}^{x} \mathbf{1} .
$$

These scalar quantities will be called the flow- and storage-based composite transfer effect indices for the system.

The flow- and storage-based composite diac effect measures and indices for the other diac transaction types can be formulated by substituting the corresponding composite diac flows and storages for the transfer flow and storage in all equations above. The simple diact effect measures and indices can also be formulated similarly by using the simple diact flows and storages instead of their composite counterparts. We will use a tilde notation for simple diact matrix measures and indices. As an example, using Table 1, the flow-based composite cycling effect index can be expressed as

$$
\mathrm{c}^{\tau}=\frac{\mathbf{1}^{T} T^{\mathrm{c}} \mathbf{1}}{\sigma^{\tau}}=\frac{\mathbf{1}^{T}\left(N-\mathcal{N}^{-1} N\right) \mathcal{N}^{-1} \mathcal{T} \mathbf{1}}{\sigma^{\tau}}
$$

Various other formulations of the cycling effect indices are presented in Section 2.9.

The input-oriented composite and simple diact effect indices can ecologically be interpreted as the direct, indirect, non-cycling, cycling, and total influence of one system compartment on another induced by all and individual environmental inputs, respectively. More specifically, the composite transfer effect index, $\mathrm{t}_{i k}$, can be interpreted as the total influence of compartment $k$ on $i$ induced by all environmental inputs. The simple transfer effect index, $\mathrm{t}_{i_{k}}$, can then be interpreted as the total influence of compartment $k$ on $i$ induced by the single environmental input, $z_{k}$. All the other input-oriented flow- and storage-based, simple and composite diact effect indices can be interpreted similarly.

The ecological interpretations of the output-oriented diact effect indices reflect the fact that the flow regime is conceptually reversed for the output-oriented analysis in the proposed framework. The output-oriented composite and simple diact effect indices can be interpreted as the direct, indirect, non-cycling, cycling, and total influence of one system compartment on another which contributes to all and individual environmental outputs, respectively. More specifically, the composite transfer effect index, $\overline{\mathrm{t}}_{i k}$, can be interpreted as the total influence of compartment $i$ on $k$ which contributes to all environmental outputs. The simple transfer effect index, $\overline{\mathrm{t}}_{i_{k}}$, can then be interpreted as the total influence of compartment $i$ on $k$ which contributes to the single environmental output, $y_{k}$. All the other output-oriented flow- and storagebased diact effect indices can be interpreted similarly.

The input-oriented composite transfer effect vector $\check{t}$ can ecologically be interpreted as the transfer effect of the system on the compartments and $\hat{t}$ as those of 
the compartments on the system. The input-oriented simple transfer effect vector $\check{\tilde{t}}$ can be interpreted as the transfer effect of the system on the compartments and $\hat{\tilde{t}}$ as those of the compartments on the subsystems. All the other input-oriented, flow- and storage-based, simple and composite diact effect vectors can be formulated as their transfer counterparts given in Eq. 2.31 by the corresponding substitutions and interpreted accordingly. The input-oriented flow- and storage-based, simple and composite, scalar diact system effect indices can also be formulated as their transfer counterparts given in Eq. 2.32 by the corresponding substitutions and interpreted as the diact effects of the system on itself.

In static ecological network analyses, the flow-based cycling index (FCI) is a standard measure quantifying cycling system flows [21]. There are also static storagebased cycling indices proposed in the literature [46, 17, 34]. The dynamic effect indices for both cycling flow and storage have recently been introduced for the first time by [12]. The static versions of these indices are introduced above in this section. The proposed simple cycling storage effect index is equivalent to the one proposed by [34], SCI, except the fact that the residence times in the authors' formulation are approximated through agent-based computational simulations, unlike the analytical formulation of residence times in the proposed methodology. The proposed index is different from the other formulations cited above. Since the simple cycling flow distribution factor, $n_{i_{i}}^{\mathrm{c}}=\left(n_{i_{i}}-1\right) / n_{i_{i}}$, is equivalent to the cycling efficiency in the FCI formulation, the first equality for $c^{\tau}$ in terms of $\mathcal{N}$ in Eq. 2.39 is equivalent to the FCI of the ecological network theory. That is, although derived with a different rationale, the proposed input-oriented, flow-based, compartmental, simple cycling flow and storage effect indices are equivalent to the FCI and SCI, respectively, as demonstrated in Case Study 3.1.

The indirect effects are considered to be flow contributions carried by subsequent steps after the first entrance into a compartment in static ecological network analyses. Even the direct transactions between compartments, after the first step, are considered as indirect contributions in various formulations proposed in the literature, as discussed in Section 2.2 [39, 41, 23, 54, 4, 33]. These indirect effect indices are formulated without actually defining the indirect flow between two system compartments. The validity and convergence of these formulations and related concepts were also discussed in the literature [47, 32, 42, 28]. The proposed indirect effect indices have different derivation rationale than these current static indices, as the proposed indices are based on the indirect flows and storages introduced in Section 2.3 and are measurable physical quantities. The proposed indirect effect indices capture experimental system behavior more accurately than the existing formulations, as presented in Case Study 3.2.

The output-oriented diact effect measures and indices can be formulated in parallel to their input-oriented counterparts developed in this section.

2.7. The diact utility measures and indices. A direct utility index was introduced in the literature for static systems [43, 18, 19] motivated by a methodology introduced by [54]. The local, compartmental normalization in this formulation makes the physical interpretation of the utility index harder as a system measure. Moreover, the geometric series expansion in the definition of the authors' utility or impact matrix formulations lack of physical meaning as discussed above and in Section 2.2. A new static direct utility index is introduced below in this section based on the dynamic diact utility index formulation proposed by [12]. This new index is different from the authors' static formulation due to its global normalization procedure in accor- 
dance with the diact effect index formulations introduced in Sec. 2.6. Therefore, the proposed direct effect index allows for local interpretations of intercompartmental dynamics relative to the entire system.

All the other novel diact utility measures and indices are also systematically introduced in this section. In general terms, the static diact utility measure will be defined as the relative diact effect of one compartment on another. The subcompartmental level formulations in parallel are straightforward, by using subflows and associated substorages instead of flows and storages.

We will first define the transfer utility measures and indices. The flow- and storage-based composite transfer utility indices of one set of compartments $K$ to another set $I, \mathbb{t}_{I K}^{\tau}$ and $\mathbb{t}_{I K}^{x}$, quantify the relative flow- and storage-based transfer effects of $K$ on $I$. They measure the normalized relative net benefit $\left(\mathbb{t}_{I K}^{\tau}>0\right.$ and $\left.\mathbb{t}_{I K}^{x}>0\right)$ or harm $\left(\mathbb{t}_{I K}^{\tau}<0\right.$ and $\left.\mathbb{t}_{I K}^{x}<0\right)$, that is transmitted from the set of compartments $K$ to $I$ based on their respective net gains (inflows and associated storages) or losses (outflows and associated storages). The indices are formulated as follows:

$$
\mathbb{t}_{I K}^{\tau}=\mathrm{t}_{I K}^{\tau}-\mathrm{t}_{K I}^{\tau} \quad \text { and } \quad \mathbb{t}_{I K}^{x}=\mathrm{t}_{I K}^{x}-\mathrm{t}_{K I}^{x}
$$

where $I, K \subseteq\{1, \ldots, n\}$. If the sets $I$ and $K$ have one element, that is, $I=\{i\}$ and $K=\{k\}$, the transfer utility indices will be denoted by $\mathbb{t}_{i k}^{\tau}$ and $\mathbb{t}_{i k}^{x}$. Note that since the transfer effect indices are dimensionless, the transfer utility indices are also dimensionless.

The flow- and storage-based composite transfer utility matrix measures are denoted by $\mathbb{T}^{\tau}=\left(\mathbb{t}_{i k}^{\tau}\right)$ and $\mathbb{T}^{x}=\left(\mathbb{t}_{i k}^{x}\right)$ and formulated in matrix form as

$$
\mathbb{T}^{\tau}=\frac{1}{\sigma^{\tau}}\left(T^{\mathrm{t}}-T^{\mathrm{t} T}\right) \quad \text { and } \quad \mathbb{T}^{x}=\frac{1}{\sigma^{x}}\left(X^{\mathrm{t}}-X^{\mathrm{t} T}\right)
$$

where the superscript $(T)$ stands for the matrix transpose. The flow-based composite transfer utility of the system on the compartments, $\check{\mathbb{t}}^{\tau}$, and that of the compartments on the system, $\hat{\mathbb{t}}^{\tau}$, will be defined as vector measures:

$$
\check{\mathbb{t}}^{\tau}=\mathbb{T}^{\tau} \mathbf{1} \text { and } \quad \hat{\mathbb{t}}^{\tau}=\mathbf{1}^{T} \mathbb{T}^{\tau} \quad \text { with } \quad \check{\mathbb{t}}^{\tau}=-\left(\hat{\mathbb{t}}^{\tau}\right)^{T} .
$$

The storage-based composite transfer utility vectors can be defined similarly. Note that, since $\mathbb{T}^{\tau}$ and $\mathbb{T}^{x}$ are skew-symmetric matrices, the flow- and storage-based composite transfer utility indices for the system are zero:

$$
\mathbb{t}^{\tau}=\mathbf{1} \mathbb{T}^{\tau} \mathbf{1}=0 \quad \text { and } \quad \mathbb{t}^{x}=1 \mathbb{T}^{x} \mathbf{1}=0 .
$$

These relationships are true for all diact utility matrices.

The flow- and storage-based composite diac utility measures and indices for the other diac transaction types can be formulated by substituting the corresponding diac flows and storages for their transfer counterparts in all equations above. The simple diact utility measures and indices can also be formulated similarly by using the simple diact flows and storages instead of their composite counterparts. We will use a tilde notation for simple diact matrix measures and indices.

The input-oriented composite and simple diact utility indices can ecologically be interpreted as the relative direct, indirect, non-cycling, cycling, and total influence of one system compartment on another induced by all and individual environmental inputs, respectively. More specifically, the composite transfer utility index, $\mathbb{t}_{i k}$, can be interpreted as the relative total influence of compartment $k$ on $i$ induced by all 
environmental inputs. The simple transfer utility index, $\mathbb{t}_{i_{k}}$, can then be interpreted as the relative total influence of compartment $k$ on $i$ induced by only the two corresponding environmental inputs, $z_{k}$ and $z_{i}$. The input-oriented flow- and storage-based diact utility indices can be interpreted similarly.

Considering the fact that the flow regime is conceptually reversed for outputoriented analysis in the context of the proposed methodology, the output-oriented diact utility indices can be interpreted accordingly. More specifically, the composite transfer utility index, $\overline{\mathbb{t}}_{i k}$, can be interpreted as the relative total influence of compartment $i$ on $k$ which contributes to all environmental outputs. The simple transfer utility index, $\overline{\mathbb{t}}_{i_{k}}$, can then be interpreted as the relative total influence of compartment $i$ on $k$ which contributes only to the two corresponding environmental outputs, $y_{k}$ and $y_{i}$. The output-oriented flow- and storage-based diact utility indices can be interpreted similarly.

The composite transfer utility vector $\check{t}$ can be interpreted as the relative transfer effect of a system on its compartments and $\hat{t}$ as those of the compartments on the system. The simple transfer utility vectors can be interpreted similarly. The diac utility vectors can be formulated as their transfer counterparts given in Eq. 2.36 by the corresponding substitutions and interpreted similarly.

The output-oriented diact utility measures and indices can be formulated in parallel to their input-oriented counterparts developed in this section.

2.8. The diact residence times. The dynamic exposure time or residence time has recently been introduced by [11] for dynamic nonlinear ecological systems. The static residence time measure will be introduced in this section. This novel system measure can find use in radiobiology, toxicology, pharmacokinetics, and other applied environmental and medical fields.

The residence time of the storage in compartment $i$ will be formulated as follows:

$$
r_{i}=\frac{x_{i}}{\tau_{i}}=\frac{x_{i_{i}}}{\tau_{i_{i}}}=\frac{x_{i_{k}}}{\tau_{i_{k}}}=\frac{s_{i_{k}}}{n_{i_{k}}}
$$

for $i=1, \ldots, n$, when $\tau_{i_{k}} \neq 0$. Note that the unit of the residence time is time, [t]. The residence time of substorage in subcompartment $i_{k}$ is the same for any $k=0, \ldots, n$, as indicated in Eq. 2.37. Therefore, the residence time matrix, $\mathcal{R}=\operatorname{diag}\left(\left[r_{1}, \ldots, r_{n}\right]\right)$, is an $n \times n$ diagonal matrix. Excluding the initial subsystem, $\mathcal{R}$ can be expressed in the following matrix forms:

$$
\mathcal{R}=\mathcal{X} \mathcal{T}^{-1}=\mathcal{X}_{k} \mathcal{T}_{k}^{-1}=X T^{-1}=S N^{-1},
$$

as formulated in Eqs. 2.22 and 2.23. It is worth noting that the residence time of the transient and diact storages in compartment $i$ or subcompartment $i_{k}$ are also equal to $r_{i}$, due to the equivalence of the outward flow and throughflow intensities [13]. From a different, probabilistic perspective, the residence time is sometimes called the turnover time [15].

The $i^{t h}$ diagonal entry of $\mathcal{R}, r_{i}$, can be interpreted as time required for generation by inward throughflow, $\tau_{i}$, of compartmental storage, $x_{i}$, in initially empty compartment $i$. Equivalently, $r_{i}$ represents the time required for outward throughflow, $\tau_{i}$, to completely empty compartment $i$ with initial storage $x_{i}$. The diagonal structure of the residence time matrix indicates that all subcompartments of compartment $i$ vanish simultaneously.

Ecologically, $\mathcal{R}$ can be used an ecosystem indicator that represents the compartmental activity levels in ecological networks: the smaller the residence time, the more active the compartments. 
2.9. Output-oriented system measures and indices. The output-oriented system analysis is discussed in Section 2.2. The output-oriented measures and indices can similarly be formulated in parallel to their input-oriented counterparts as formulated in the present manuscript. We will introduce only the output-oriented simple cycling effect indices below, as an example.

The input- and output-oriented simple cycling flow and storage matrices are listed in Tables 1 and 2. Alternative formulations for the simple cycling flows and storages are also proposed by [14]. The input- and output- oriented simple flow and storage distribution matrices in this alternative formulation are the same: $\mathcal{N}^{c}=\overline{\mathcal{N}}^{c}=(\mathcal{N}-$ I) $\mathcal{N}^{-1}$ and $\mathcal{S}^{c}=\overline{\mathcal{S}}^{c}=\mathcal{R} \mathcal{N}^{c}$. Therefore, the input- and output-oriented simple cycling effect indices can be expressed in the following various forms:

$$
\begin{aligned}
& \tilde{\mathrm{c}}^{\tau}=\frac{\mathbf{1}^{T}(\mathcal{N}-I) \mathcal{N}^{-1} \mathcal{T} \mathbf{1}}{\sigma^{\tau}}=\frac{\mathbf{1}^{T}\left(\mathcal{T}-\mathcal{S}^{-1} \mathcal{X}\right) \mathbf{1}}{\sigma^{\tau}}=\tilde{\overline{\mathrm{c}}}^{\tau}, \\
& \tilde{\mathrm{c}}^{x}=\frac{\mathbf{1}^{T}(\mathcal{N}-I) \mathcal{N}^{-1} \mathcal{X} \mathbf{1}}{\sigma^{x}}=\frac{\mathbf{1}^{T}\left(I-\mathcal{R} \mathcal{S}^{-1}\right) \mathcal{X} \mathbf{1}}{\sigma^{x}}=\tilde{\overline{\mathrm{c}}}^{x} .
\end{aligned}
$$

The second equalities for both $\tilde{\mathrm{c}}^{\tau}$ and $\tilde{\mathrm{c}}^{x}$ are obtained by using the fact that

$$
\mathcal{S}=\mathcal{R} \mathcal{N} \Rightarrow \mathcal{N}^{-1}=\mathcal{S}^{-1} \mathcal{R} \text { and } \quad \mathcal{X}=\mathcal{R} \mathcal{T} \Rightarrow \mathcal{T}^{-1}=\mathcal{X}^{-1} \mathcal{R}
$$

The equivalence of the input- and output-oriented simple cycling distribution matrices implies that of the compartmental effect vectors. The corresponding effect vectors for subsystems, however, are different. That is, since

$$
\tilde{\tau}^{\mathrm{c}}=\tilde{\bar{\tau}}^{\mathrm{c}} \quad \text { and } \quad \tilde{x}^{\mathrm{c}}=\tilde{\bar{x}}^{\mathrm{c}}
$$

as shown by [14], we have

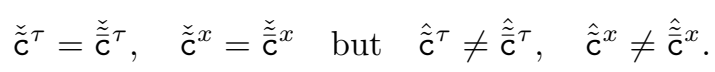

This relationship indicates that the amount of environmental inputs cycling in each compartment within the system are the same in both input- and output-orientations.

2.10. Quantitative definitions of interspecific interactions. The flow of energy and matter through ecosystems is one of the main topics of ecosystem ecology. The proposed methodology is particularly designed for quantitative analysis of ecosystem flows and storages. The method is applicable to both naturogenic and anthropogenic ecological models. The physical terminology reflecting this generality and wide applicability of the proposed method can be adjusted depending on specific ecological models of interest.

One of the immediate potential ecological applications of the method is the quantitative analysis of food chains, food webs, and interspecific interactions within the webs. In the context of the proposed methodology, a food web can be considered as a naturogenic ecosystem, the system compartments represent species in the food web, the conserved quantity in question becomes nutrient or energy, and flow paths correspond to food chains. All formulations and relationships introduced in physical terms in the present paper are applicable to food webs and can, therefore, be translated to the food web terminology using these and similar correspondences and can be interpreted accordingly.

A quantitative technique for the classification of interspecific interactions within food webs has recently been developed based on the diact flows [14]. We will reformulate this approach in terms of the effect and utility indices developed in the 
present manuscript and also propose a novel quantitative characterization for main types of interspecific interactions in this section. As it uses system flows for characterization of interspecific interactions, this quantitative technique sets up a bridge between ecosystem ecology and community ecology.

Community ecology studies interaction types among species of food webs. In general, the interspecific interactions are structurally determined by the network topology without regard for system flows $[37,36]$. Increasing complexity of intricate food webs in most cases disallows structural determination due to various factors, such as multiple food chains of possibly different lengths between two species [25, 62, 7]. A parametric classification is attempted through a utility analysis in the literature, but it has disadvantages due to the geometric series expansion used in the formulation of indirect contributions, as discussed in Section $2.2[54,44,18,16,53]$. Moreover, unlike some of these authors' claims, some interactions, such as amensalism, cannot be classified through the flow analysis, as explained below.

We will first reiterate the flow-based classification technique for the interspecific interactions recently proposed by [14]. The diact flows can be used for the quantitative characterization of the interspecific interactions within food webs. Since the diact transactions quantify nutrient and energy transfers within food webs, nontrophic interactions that are not part of a food web cannot be categorized through the proposed technique. For example, amensalism is defined as an interaction where an organism inflicts harm to another organism without any effect received by itself, such as some common mould species producing antibiotics as a waste product that kill bacteria in their microenvironments. Since there is no nutrient or energy transfer involved in this process, such nontrophic interactions need to be determined and classified topologically or qualitatively.

The flow transfers between two species can be grouped into the natural binary categories of net positive transfer or no transfer. These categories are used to quantitatively define the corresponding binary categories of interspecific interactions: neutralism and antagonism. Neutralism will be described as the theoretical, base-state where two species do not affect each other. Antagonism can then be defined as the interaction of two species that is beneficial for one partner, "predator", but detrimental for the other one, "prey". Being beneficial and detrimental in this description will quantitatively be defined as net positive and negative diact flow transmission from one species to another. Depending on the diact transaction type, the corresponding diact interspecific interactions will be categorized as directly, indirectly, acyclically, cyclically, and totally neutral or antagonistic interactions.

This quantitative technique including sign and strength analysis components will be extended to main types of interspecific interactions in this section. The sign analysis determines the neutral and antagonistic nature of the interactions - whether the interaction is beneficial or harmful to the species involved. Since the proposed methodology can quantitatively determine the net benefit received by the involved species from each other, the strength analysis quantifies the strength of these interactions. This determination is important in distinguishing, for example, parasitism, which mainly reduce fitness of host species, from predation. The sign and strength of the direct interaction between species $i$ and $j$ induced by environmental inputs will be defined as

$$
\delta_{i j}^{\mathrm{d}}=\operatorname{sgn}\left(\tau_{i j}^{\mathrm{d}}-\tau_{j i}^{\mathrm{d}}\right)=\operatorname{sgn}\left(\mathbb{d}_{i j}^{\tau}\right) \quad \text { and } \quad \mu_{i j}^{\mathrm{d}}=\frac{\left|\tau_{i j}^{\mathrm{d}}-\tau_{j i}^{\mathrm{d}}\right|}{\tau_{i j}^{\mathrm{d}}+\tau_{j i}^{\mathrm{d}}}
$$

respectively, where $\operatorname{sgn}(\cdot)$ is the sign function. In community ecology, the positive 
TABLE 3

Quantitative definitions of interspecific interactions.

\begin{tabular}{|c|c|c|c|c|}
\hline Type & \multicolumn{3}{|c|}{ Definition } & Strength \\
\hline Neutralism & $\mathbb{d}_{i j}^{\tau}=0$ & $\mathrm{~d}_{i k}^{\tau} \mathrm{d}_{j k}^{\tau}=0$ & $\dot{\mathrm{i}}_{i j}^{\tau}=0$ & $\mu_{i j}^{n}=0$ \\
\hline Mutualism & $\mathbb{d}_{i j}^{\tau}=0$ & $\mathrm{~d}_{i k}^{\tau} \mathrm{d}_{j k}^{\tau}=0$ & $\dot{\mathrm{i}}_{i j}^{\tau} \neq 0$ & $\mu_{i j}^{m}=\left(\tau_{i j}^{\mathrm{i}}+\tau_{j i}^{\mathrm{i}}\right) /\left(\tau_{j}+\tau_{i}\right)$ \\
\hline Commensalism & $\mathbb{d}_{i j}^{\tau}=0$ & $\mathrm{~d}_{i k}^{\tau} \mathrm{d}_{j k}^{\tau} \neq 0$ & $\mu_{i j, k}^{c} \gg 1 / 2$ & $\left|\tau_{j k}^{\mathrm{d}}-\tau_{j k}^{\mathrm{d}}\right| /\left(\tau_{j k}^{\mathrm{d}}+\tau_{j k}^{\mathrm{d}}\right.$ \\
\hline Competition & $\mathbb{d}_{i j}^{\tau}=0$ & $\mathrm{~d}_{i k}^{\tau} \mathrm{d}_{j k}^{\tau} \neq 0$ & $\mu_{i j, k}^{c} \ll 1 / 2$ & $\mu_{i j, k}={ }_{e} \tau_{i k}$ \\
\hline Exploitation & $\mathrm{d}_{i j}^{\tau}>0$ & $\mathrm{~d}_{j i}^{\tau}=0$ & & $\mu_{i j}^{e}=\tau_{i j}^{\mathrm{d}} / \tau_{j}$ \\
\hline
\end{tabular}

and negative effects of one species on another are represented by $(+)$ and $(-)$ signs. Following the convention, instead of $(+1)$ and $(-1),(+)$ and $(-)$ notations will be used for the sign of the diact interactions. The strength, $\mu_{i j}^{\mathrm{d}}$, is defined to be zero, if both terms in its denominator are zero. Note that $0 \leq \mu_{i j}^{\mathrm{d}} \leq 1$.

Multiple other formulations of the strength for the analysis of diact interactions at different scales are introduced by [14]. At the global scale, the sign and strength of the local direct interactions between species $i$ and $j$ induced by environmental inputs, for example, can accordingly be formulated as

$$
\delta_{i j}^{\mathrm{d}}=\operatorname{sgn}\left(\mathbb{d}_{i j}^{\tau}\right) \quad \text { and } \quad \mu_{i j}^{\mathrm{d}}=\frac{\left|\tau_{i j}^{\mathrm{d}}-\tau_{j i}^{\mathrm{d}}\right|}{\sigma^{\tau}}=\left|\mathbb{d}_{i j}^{\tau}\right|
$$

using the utility indices.

The direct neutral relationship between species $i$ and $j$ and "predation" of species $i$ on $j$ can quantitatively be characterized, respectively, as follows:

$$
\mathbb{d}_{i j}^{\tau}=0 \Rightarrow \delta_{i j}^{\mathrm{d}}=(0) \quad \text { and } \quad \mathbb{d}_{i j}^{\tau}>0 \Rightarrow \delta_{i j}^{\mathrm{d}}=(+) .
$$

The sign and strength of the other diact interactions, as well as their characterization can be formulated similarly by using the corresponding diact flows in Eqs. 2.43 and 2.45 instead of the direct flows.

The strength and sign of the diact interactions between species $i$ and $j$ are then formulated as

$$
\mu_{i j}^{*}=\left(\mu_{i j}^{\mathrm{d}}, \mu_{i j}^{\mathrm{i}}, \mu_{i j}^{\mathrm{a}}, \mu_{i j}^{\mathrm{c}}, \mu_{i j}^{\mathrm{t}}\right) \quad \text { and } \quad \delta_{i j}^{*}=\left(\delta_{i j}^{\mathrm{d}}, \delta_{i j}^{\mathrm{i}}, \delta_{i j}^{\mathrm{a}}, \delta_{i j}^{\mathrm{c}}, \delta_{i j}^{\mathrm{t}}\right) .
$$

The elements of the ordered quintuples $\delta_{i j}^{*}$ and $\mu_{i j}^{*}$ represent the neutral and antagonistic nature of the diact interactions and their strength, respectively. As an example, $\delta_{i j}^{*}=(0,+,-,+,+)$ indicates that the interactions between $i$ and $j$ are directly neutral, indirectly, acyclically, cyclically, and totally antagonistic. Moreover, the antagonistic interactions are indirectly, cyclically, and totally beneficial but acyclically detrimental to species $i$.

The standard qualitative definitions for the main types of interspecific interactions can easily be found in the literature, even in textbooks [25, 62]. We will propose a quantitative characterization for these interspecific interactions within a food web below. It is worth noting, however, that this characterization is not exclusive. That is, there may be special cases in nature that require modified quantitative definitions. In the following definitions, for example, intraguild predation is not included in the quantitative definition of competition.

Neutralism: A theoretical base-state where species species $i$ and $j$ do not directly or indirectly affect each other, that is, $\mathbb{d}_{i j}^{\tau}=0$ and $\dot{\mathrm{i}}_{i j}^{\tau}=0$. They also do 
not share a common resource: $\mathrm{d}_{i k}^{\tau} \mathrm{d}_{j k}^{\tau}=0$ for any species $k$. The strength of neutralism is defined to be $\mu_{i j}^{n}=0$.

Mutualism: Species $i$ and $j$ have no direct transactions but have indirect interactions through other species of the food web, that is, $\mathbb{d}_{i j}^{\tau}=0$ and $\dot{\mathrm{i}}_{i j}^{\tau} \neq 0$. They also do not share a common resource: $\mathrm{d}_{i k}^{\tau} \mathrm{d}_{j k}^{\tau}=0$ for any species $k$. The strength of mutualism is defined as $\mu_{i j}^{m}=\left(\tau_{i j}^{\mathrm{i}}+\tau_{j i}^{\mathrm{i}}\right) /\left(\tau_{i}+\tau_{j}\right)$.

Commensalism: Species $i$ and $j$ have no direct transactions but they both have disproportionate direct inflow from a common resource: $\mathbb{d}_{i j}^{\tau}=0, \mathrm{~d}_{i k}^{\tau} \mathrm{d}_{j k}^{\tau} \neq 0$, and $\mu_{i j, k}^{c} \gg 1 / 2$ for some species $k$ where the strength of commensalism is defined as $\mu_{i j, k}^{c}=\left|\tau_{i k}^{\mathrm{d}}-\tau_{j k}^{\mathrm{d}}\right| /\left(\tau_{i k}^{\mathrm{d}}+\tau_{j k}^{\mathrm{d}}\right)$.

Competition: Species $i$ and $j$ have typically no direct transactions but they both have proportionate direct inflows from the same resource: $\mathbb{d}_{i j}^{\tau}=0, \mathrm{~d}_{i k}^{\tau} \mathrm{d}_{j k}^{\tau} \neq 0$, and $\mu_{i j, k}^{c} \ll 1 / 2$ for some species $k$ where the strength of competition, $\mu_{i j, k}^{c}$, is the same as that of commensalism.

Exploitation: (Predation/Grazing/Parasitism) The antagonistic interaction of species $i$ and $j$ that is beneficial for species $i$, "predator," but detrimental for species $j$, "prey": $\mathrm{d}_{i j}^{\tau}>0$ and $\mathrm{d}_{j i}^{\tau}=0$. The strength of exploitation is $\mu_{i j}^{e}=\tau_{i j}^{\mathrm{d}} / \tau_{j}$. Parasitism can also be defined similar to predation except that the strength of the interactions of parasite species $i$ with the host species $j$ are all small, that is, $\mu_{i j}^{e} \approx 0$ for all $j$.

The strength of mutualism and exploitation can also be reformulated for the analysis of interspecific interactions at the global scale as follows:

$$
\mu_{i j}^{m}=\frac{\tau_{i j}^{\mathrm{i}}+\tau_{j i}^{\mathrm{i}}}{\sigma^{\tau}}=\mathbf{i}_{i j}^{\tau}+\mathbf{i}_{j i}^{\tau} \quad \text { and } \quad \mu_{i j}^{e}=\frac{\tau_{i j}^{\mathrm{d}}}{\sigma^{\tau}}=\mathrm{d}_{i j}^{\tau}
$$

using the effect indices.

The flow-based quantitative definitions of the main interspecific interactions and their strength are listed in Table 3. The storage-based quantitative definitions of interspecific interactions can be formulated in parallel by substituting the corresponding diact storages instead of the diact flows in the descriptions above. The storage-based formulations represent the history of interspecific interactions while the flow-based formulations represent simultaneous interactions. For classification of the interspecific interactions induced by individual environmental inputs, the simple diact flows and storages can be used instead of their composite counterparts. Applications of the proposed quantitative characterization and classification are presented in the case studies.

3. Results. The proposed methodology is applied to two ecological network models from the literature in this section. The measures and indices for the diact effects, utilities, and residence times introduced in this manuscript together with their ecological implications are presented for these models.

The results indicate that the proposed methodology precisely quantifies ecosystem functions, properties, and behaviors, effectively determine the environmental influence on system compartments and intercompartmental dynamics, and, thus, can be used for rigorous analysis of ecosystems. Although the models are extensively studied in the literature, some of these results have not yet been reported. It is worth emphasizing, however, that this present work proposes a new mathematical method - a systematic technique designed for analyzing static ecological network models using the proposed measures and indices as ecosystem indicators - and it is not itself a model. Therefore, we focus more on demonstrating the efficiency and wide applicability of the mathe- 


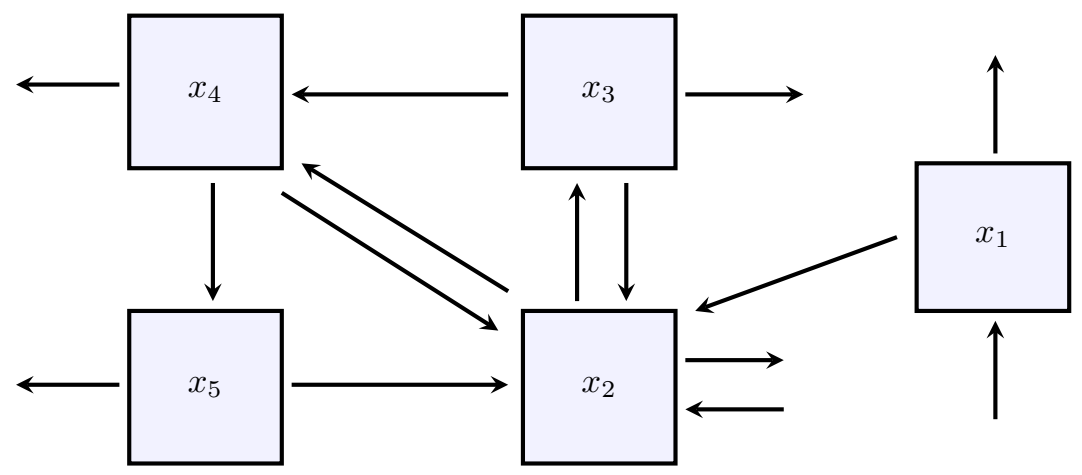

FIG. 4. Schematic representation of the flow regime of the Cone Spring ecosystem model (Case Study 3.1).

matical system analysis tools introduced as quantitative ecological indicators in the present paper. It is expected that once the method is accessible to a broader community of environmental ecologists, it can be used for ecological inferences and detailed analyses of specific models of interest.

3.1. Case study. The Cone Spring ecosystem model is a commonly studied ecological network, first proposed by [52]. The model has recently been analyzed through the proposed methodology by [14]. In particular, the substorage and subthroughflow matrix measures and the transient and diact flows and storages are presented. In this case study, the measures and indices for the diact effects, utilities, and residence times are presented for this model.

Cone Spring is a small, shallow spring-brook located in Louisa County, Iowa. The study area consists of $116 \mathrm{~m}^{2}$. The network has 5 compartments representing 1-plants, 2-detritus, 3-bacteria, 4-detritus feeders, and 5-carnivores. These compartments are connected by the transaction of energy between them. Therefore, the conserved quantity in question is energy for this ecosystem model. The system flow regime is given as follows:

$$
F=\left[\begin{array}{ccccc}
0 & 0 & 0 & 0 & 0 \\
8881 & 0 & 1600 & 200 & 167 \\
0 & 5205 & 0 & 0 & 0 \\
0 & 2309 & 75 & 0 & 0 \\
0 & 0 & 0 & 370 & 0
\end{array}\right], \quad y=\left[\begin{array}{c}
2303 \\
3969 \\
3530 \\
1814 \\
203
\end{array}\right], \quad z=\left[\begin{array}{c}
11184 \\
635 \\
0 \\
0 \\
0
\end{array}\right]
$$

The energy storages are $x=[285,3579.4,116.6,60,17]^{T}[45]$. The units for energy flows and storages are $\left(\mathrm{kkal} \mathrm{m}^{-2} \mathrm{y}^{-1}\right)$ and $\left(\mathrm{kkal} \mathrm{m}^{-2}\right)$, respectively.

The system partitioning methodology produces the subthroughflow matrix as formulated in Eq. 2.14 as follows:

$$
T=\left[\begin{array}{ccccc}
11184 & 0 & 0 & 0 & 0 \\
10717 & 766 & 0 & 0 & 0 \\
4858 & 347 & 0 & 0 & 0 \\
2225 & 159 & 0 & 0 & 0 \\
345 & 25 & 0 & 0 & 0
\end{array}\right]
$$

Since there is no environmental input into the $3^{\text {rd }}, 4^{\text {th }}$, and $5^{\text {th }}$ compartments $\left(z_{3}=\right.$ 
$z_{4}=z_{5}=0$ ), the $3^{r d}, 4^{\text {th }}$, and $5^{\text {th }}$ subsystems represented on the corresponding columns of $T$ (and of $X$ ) are null. That is, there is no subthroughflow at (and, therefore, no substorage in) the corresponding subcompartments of these subsystems.

The subsystem partitioning methodology yields the diact energy flows and storages as listed in Table 1. The composite direct flow matrix is $T^{d}=F$. The composite indirect flow and storage distribution matrices are

$$
N^{\mathrm{i}}=\left[\begin{array}{ccccc}
0 & 0 & 0 & 0 & 0 \\
0.1641 & 0.1713 & 0.0121 & 0.0950 & 0.0839 \\
0.4343 & 0 & 0.1448 & 0.0811 & 0.2426 \\
0.1989 & 0.0065 & 0.0643 & 0.0371 & 0.1111 \\
0.0309 & 0.0323 & 0.0122 & 0 & 0.0173
\end{array}\right] \text { and } S^{\mathrm{i}}=\mathcal{R} N^{\mathrm{i}}
$$

where the residence time matrix is $\mathcal{R}=\operatorname{diag}([0.0255,0.3117,0.0224,0.0252,0.0460])$. Therefore, the composite indirect flow matrix, as listed in Table 1, becomes

$$
T^{\mathrm{i}}=N^{\mathrm{i}} \mathcal{T}=\left[\begin{array}{ccccc}
0 & 0 & 0 & 0 & 0 \\
1835.74 & 1967.00 & 63.02 & 226.41 & 31.04 \\
4857.67 & 0 & 753.81 & 193.28 & 89.76 \\
2224.91 & 75.00 & 334.40 & 88.52 & 41.11 \\
345.31 & 370.00 & 63.53 & 0 & 6.38
\end{array}\right]
$$

where $\mathcal{T}=\operatorname{diag}([11184,11483,5205,2384,370])$. The associated composite energy storages generated by these indirect flows are

$$
X^{\mathrm{i}}=\left[\begin{array}{ccccc}
0 & 0 & 0 & 0 & 0 \\
572.22 & 613.14 & 19.65 & 70.58 & 9.68 \\
108.81 & 0 & 16.89 & 4.32 & 2.01 \\
56.0 & 1.89 & 8.42 & 2.23 & 1.03 \\
15.87 & 17.0 & 2.91 & 0 & 0.29
\end{array}\right]
$$

The composite acyclic energy flow and storage matrices, $T^{\mathrm{a}}$ and $X^{\mathrm{a}}$, can also be computed as given in Table 1. It can be shown that $\tau_{54}^{\mathrm{a}} / \tau_{4}=0.152$ and $x_{54}^{\mathrm{a}} / x_{4}=$ 0.278. In other words, the energy flow and storage transmitted from the detritus feeders to the carnivores that do not cycle after being transmitted are $15.2 \%$ and $27.8 \%$ of the outward energy throughflow at and storage in the detritus feeders, respectively. Since $27.8 \%$ of the energy storage in the detritus feeders is transferred to the carnivores, the ten percent energy transfer law from one trophic level to the next, first introduced by [31], does not seem to be an accurate estimate for this food web. As this result illustrates, the proposed methodology constructs a quantitative base for testing empirical ecological hypotheses and, potentially, theoretical developments.

The flow-based simple cycling and acyclic effect matrix measures for the Cone Spring ecosystem model are also given below:

$$
\tilde{\mathrm{C}}^{\tau}=\left[\begin{array}{ccccc}
0 & 0 & 0 & 0 & 0 \\
0.0599 & 0.0043 & 0 & 0 & 0 \\
0.0230 & 0.0016 & 0 & 0 & 0 \\
0.0027 & 0.0002 & 0 & 0 & 0 \\
0.0002 & 0.0 & 0 & 0 & 0
\end{array}\right] \text { and } \tilde{\mathrm{A}}^{\tau}=\left[\begin{array}{ccccc}
0 & 0 & 0 & 0 & 0 \\
0.29 & 0 & 0 & 0 & 0 \\
0.1356 & 0.0097 & 0 & 0 & 0 \\
0.0699 & 0.0050 & 0 & 0 & 0 \\
0.0111 & 0.0008 & 0 & 0 & 0
\end{array}\right] \text {. }
$$

Note that, the $(i, k)$-elements of $\tilde{\mathrm{C}}^{\tau}$ and $\tilde{\mathrm{A}}^{\tau}$ indicate the cycling and non-cycling effects of compartment $k$ on $i$, induced by single environmental input $z_{k}$. The $(i, k)$-elements 
of $\tilde{\mathrm{C}}^{\tau}$ can also be interpreted as indirect influence of subcompartment $i_{k}$ on itself. For example, $(2,1)$-element of $\tilde{\mathrm{C}}^{\tau}, \mathrm{c}_{2_{1}}^{\tau}=0.0599$, implies that $5.9 \%$ of total system throughflow, $\sigma^{\tau}=30626 \mathrm{kkal} \mathrm{m}^{-2} \mathrm{y}^{-1}$, is cycling at subcompartment $2_{1}$. That is, the energy flow segment of $\mathrm{c}_{2_{1}}^{\tau} \sigma^{\tau}=1835.7 \mathrm{kkal} \mathrm{m}^{-2} \mathrm{y}^{-1}$ is cycling within the energy throughflow, $\tau_{2_{1}}=10717 \mathrm{kkal} \mathrm{m}^{-2} \mathrm{y}^{-1}$, generated at the detritus compartment by the environmental energy input into the plants, $z_{1}=11184 \mathrm{kkal} \mathrm{m}^{-2} \mathrm{y}^{-1}$. This is the maximum subcompartmental simple cycling energy flow within the system. The non-cycling segment of the same throughflow is $\mathrm{a}_{2_{1}}^{\tau} \sigma^{\tau}=8881 \mathrm{kkal} \mathrm{m}^{-2} \mathrm{y}^{-1}$. Note that, $\left(\mathbf{a}_{2_{1}}^{\tau}+\mathbf{c}_{2_{1}}^{\tau}\right) \sigma^{\tau}=\tau_{2_{1}}$. All nonzero simple acyclic effect indices, $\mathbf{a}_{i_{k}}^{\tau}$, being about an order of magnitude greater than the corresponding simple cycling effect indices, $\mathrm{c}_{i_{k}}^{\tau}$, indicates that the simple acyclic effects dominate the simple cycling effects within the entire system. The ratio of the simple acyclic to the simple cycling effects of the system is $\mathrm{a}^{\tau} / \mathrm{c}^{\tau}=5.68$.

Let three sets of compartments be given as $K=\{1,2\}, J=\{3,4\}$, and $I=\{5\}$. Using Eq. 2.29, the storage-based composite transfer effects of the sets of compartments $K$ and $J$ on $I$ become

$$
\mathrm{t}_{I K}^{x}=\frac{x_{51}^{\mathrm{t}}+x_{52}^{\mathrm{t}}}{\sigma^{x}}=0.0081 \quad \text { and } \quad \mathrm{t}_{I J}^{x}=\frac{x_{53}^{\mathrm{t}}+x_{54}^{\mathrm{t}}}{\sigma^{x}}=0.0049
$$

where $X^{\mathrm{t}}$ can be computed as formulated in Table 1, and the total system energy storage based on the definition given in Eq. 2.30 is $\sigma^{x}=4058 \mathrm{kkal} \mathrm{m}^{-2}$. These results indicate that the composite transfer storage effects of the plants and detritus (compartments 1 and 2), directly and indirectly, on the carnivores is $\mathrm{t}_{I K}^{x} / \mathrm{t}_{I J}^{x}=1.65$ times more than that of the detritus feeders and bacteria (compartments 3 and 4 ). A similar computation also shows that the composite direct storage effect of the plants and detritus on the carnivores is $\mathrm{d}_{I K}^{x}=0$, and that of the detritus feeders and bacteria is $\mathrm{d}_{I J}^{x}=0.0042$. Consequently, although there is no direct effect of the plants and detritus on the carnivores, in terms of energy storage transfer, their total effects due to the indirect interactions are greater than the total effects of the detritus feeders and bacteria.

We define new vectors $\mathrm{e}^{\tau}$ and $\mathrm{e}^{x}$ as the vectors of flow- and storage-based composite diact effect indices for the system. That is,

$$
\mathrm{e}^{\tau}=\left[\mathrm{d}^{\tau}, \dot{\mathrm{i}}^{\tau}, \mathrm{a}^{\tau}, \mathrm{c}^{\tau}, \mathrm{t}^{\tau}\right]^{T} \quad \text { and } \quad \mathrm{e}^{x}=\left[\mathrm{d}^{x}, \mathrm{i}^{x}, \mathrm{a}^{x}, \mathrm{c}^{x}, \mathrm{t}^{x}\right]^{T}
$$

For the Cone Spring ecosystem, the vectors become

$$
\mathrm{e}^{\tau}=[0.61,0.44,0.84,0.22,1.06]^{T} \quad \text { and } \quad \mathrm{e}^{x}=[0.88,0.38,0.92,0.34,1.26]^{T} .
$$

These results show that the acyclic effects are larger than the cycling effects and the direct effects dominate the indirect effects, unlike the Neuse River Estuary ecosystem analyzed in the next case study. Therefore, since these scalar system indices ascertain differences in the global characteristics of ecosystems, they can be used as system indicators for ecosystem characterization.

The proposed methodology construct a base for the development of more accurate measures and indices than some of their counterparts in the literature, as discussed in Sections 2.2 and 2.6. For example, although the proposed input-oriented, flow-based, compartmental, simple cycling effect index and FCI are the same, the proposed storage-based simple cycling effect index and the storage-based cycling index 
introduced by [17], CIS, are significantly different. That is,

$$
\tilde{\mathrm{c}}^{\tau}=0.0919=\mathrm{FCI} \quad \text { and } \quad \tilde{\mathrm{c}}^{x}=0.1559 \neq 0.4433=\mathrm{CIS} .
$$

Unlike the large difference between FCI and CIS, the proposed methodology suggests, comparably, that $16 \%$ of the total system storage cycling corresponds to $9 \%$ of the total system throughflow cycling. The difference is due to the difference in the formulations of these indices. Instead of the residence time matrix, $\mathcal{R}$, a constant time interval $\Delta t$ is used by the authors in various related formulations [19, 17]. Since SCI is approximated through computationally expensive agent-based simulations, a comparison of $\tilde{\mathrm{c}}^{x}$ with SCI is not possible [34].

The storage-based composite direct and indirect utility matrix measures are given below for the Cone Spring ecosystem as formulated in Section 2.6. Using Eq. 2.35, they can be expressed as

$$
\mathbb{D}^{x}=\left[\begin{array}{ccccc}
0 & -0.6822 & 0 & 0 & 0 \\
0.6822 & 0 & 0.0941 & 0.0010 & 0.0128 \\
0 & -0.0941 & 0 & -0.0005 & 0 \\
0 & -0.0010 & 0.0005 & 0 & -0.0042 \\
0 & -0.0128 & 0 & 0.0041 & 0
\end{array}\right]
$$

and

$$
\mathbb{I}^{x}=\left[\begin{array}{ccccc}
0 & -0.141 & -0.0268 & -0.0138 & -0.0039 \\
0.141 & 0 & 0.0048 & 0.0169 & -0.0018 \\
0.0268 & -0.0048 & 0 & -0.0010 & -0.0002 \\
0.0138 & -0.0169 & 0.0010 & 0 & 0.0003 \\
0.0039 & 0.0018 & 0.0002 & -0.0003 & 0
\end{array}\right] .
$$

It can be seen that the off-diagonal elements in the second column of $\mathbb{D}^{x}$ are all negative. This implies that

$$
\mathbb{d}_{i 2}^{x}<0 \quad \Rightarrow \quad \mathrm{d}_{i 2}^{x}<\mathrm{d}_{2 i}^{x}
$$

for $i=1, \ldots, 5$. That is, the detritus gains net energy from all species through direct transactions. The elements in the second column of $\mathbb{I}^{x}$ are also negative or zero except $\mathbb{I}_{52}^{x}=0.0018>0$. This indicates that, while the carnivores lose net energy to the detritus through direct transactions as expected, it is, interestingly, the only species that gains net energy from the detritus indirectly through other species. On the other hand, because the composite transfer utility of the detritus on the carnivores is negative, that is $\mathbb{D}_{52}^{x}+\mathbb{I}_{52}^{x}=\mathbb{T}_{52}^{x}=-0.011<0$, the detritus gains net energy from the carnivores through their total, direct and indirect, interactions. Quantitatively, the net energy transferred from the carnivores and stored in the detritus is $1.1 \%$ of the total system energy storage, that is, $0.011 \sigma^{x}=44.638 \mathrm{kkal} \mathrm{m}^{-2}$.

We define new matrices whose columns are the flow- and storage-based diact

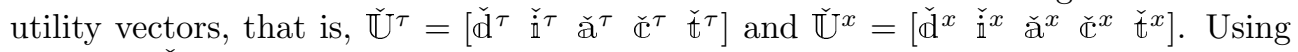
Eq. 2.36, $\check{\mathbb{U}}^{x}$ for the Cone Spring ecosystem becomes

$$
\check{\mathbb{U}}^{x}=\left[\begin{array}{ccccc}
-0.6822 & -0.1855 & -0.7223 & -0.1455 & -0.8677 \\
0.7902 & 0.1610 & 0.7849 & 0.1663 & 0.9512 \\
-0.0946 & 0.0207 & -0.0602 & -0.0137 & -0.0739 \\
-0.0048 & -0.0019 & -0.0020 & -0.0047 & -0.0066 \\
-0.0086 & 0.0057 & -0.0005 & -0.0025 & -0.0030
\end{array}\right] .
$$


The entries of the first row of $\check{\mathbb{U}}^{x}$ are negative for all diact interaction types. This implies a net energy transfer from the plants to the system in all these interaction types, which is a manifestation of the plants being the primary producer - the first trophic level of this food web. On the other hand, the positive signs of all entries on the second row indicate that the detritus gains net diact energy from the system in all interaction types. This is a reflection of the detritus being the last compartment of the food chains. The signs of the matrix entries on the first and last columns of $\check{\mathbb{U}}^{x}$ are the same. This shows that the net gain or loss of the compartments from the system are mainly determined by direct transactions. This is consistent with the observation made above that the direct effects dominate the indirect effects within the system. It can be seen from the elements of the transfer utility vector that only the detritus gains net energy from the system, that is $\check{t}_{2}^{x}=0.95>0$, and all the other compartments have a net energy loss to the system. Therefore, the total net energy stored in the detritus after being transmitted from the species in this ecosystem is $95 \%$ of the total system energy storage, that is, $0.95 \sigma^{x}=3860 \mathrm{kkal} \mathrm{m}^{-2}$.

The flow-based composite transfer utility matrix can be calculated as given in Eq. 2.35:

$$
\mathbb{T}^{\tau}=\left[\begin{array}{ccccc}
0 & -0.3499 & -0.1586 & -0.0726 & -0.0113 \\
0.3499 & 0 & -0.1157 & -0.0639 & -0.0056 \\
0.1586 & 0.1157 & 0 & -0.0071 & 0.0009 \\
0.0726 & 0.0639 & 0.0071 & 0 & -0.0107 \\
0.0113 & 0.0056 & -0.0009 & 0.0107 & 0
\end{array}\right]
$$

The other flow-based composite diact utility measures can also be obtained similarly. It can be seen that $\mathbb{t}_{54}^{\tau}>0$. This result indicates that there is a net energy flow from the detritus feeders to the carnivores through total transactions. Similar computations show that $\mathbb{d}_{54}^{\tau}>0, \dot{\mathrm{i}}_{54}^{\tau}<0, \mathrm{a}_{54}^{\tau}>0, \mathbb{C}_{54}^{\tau}>0$. Therefore, the ordered quintuples defined for quantitative classification of interspecific interactions and their strength in Section 2.10 become $\delta_{54}^{*}=(+,-,+,+,+)$ and $\mu_{54}^{*}=(1.00,1.00,0.80,0.61,0.80)$ (see Fig. 4). Consequently, all diact interactions between the carnivores and detritus feeders are antagonistic. Interestingly, the result indicates that, while the carnivores directly prey on the detritus feeders, the latter indirectly "feed" on the former. The carnivores receive net benefit from the detritus feeders also through acyclic and cycling interactions. Since the direct interactions dominate their relationship, the carnivores receive net benefit from their overall interactions. They are more antagonistic in their direct and indirect interactions $\left(\mu_{54}^{\mathrm{d}}=\mu_{54}^{\mathrm{i}}=1.00\right)$ and less so in their cycling interactions $\left(\mu_{54}^{\mathrm{c}}=0.61\right)$. That is, while the relative direct and indirect effect these species generate on each other is large, their relative cycling effect on each other is smaller. In all categories, there are strong interactions between these two species.

The quantitative technique for the characterization and classification of interspecific interactions introduced in Section 2.10 indicates that there is no neutralism, commensalism, and competition within this ecosystem. The plants are in mutualistic relationships with each of the bacteria, detritus feeders, and carnivores with the corresponding strengths of $\mu_{31}^{m}=0.30, \mu_{41}^{m}=0.16$, and $\mu_{51}^{m}=0.03$. Naturally, the strength of mutualism decreases with the length of the food chains between the plants and the others. The plants and detritus, the carnivores and detritus, the bacteria and detritus feeders, and the detritus feeders and carnivores are all linked through exploitive interactions. The second species or compartments in these pairs benefit from the first ones with the corresponding strengths of $\mu_{21}^{e}=0.79, \mu_{25}^{e}=0.45, \mu_{43}^{e}=0.01$, 
and $\mu_{54}^{e}=0.16$. The detritus can be excluded from this classification for being an abiotic compartment of this ecosystem. It can be seen from the strength analysis that the exploitive interaction between the detritus feeders and bacteria is less detrimental to the bacteria than that between the detritus feeders and carnivores to the detritus feeders. As described in Section 2.10, although the strength of the exploitive interaction between the detritus feeders and bacteria is small, the detritus feeders cannot be considered as parasites because the strength of their interaction with another compartment, the detritus, is large, $\mu_{42}^{e}=0.20$.

For the Cone Spring ecosystem, the residence time matrix introduced in Section 2.8 becomes

$$
\mathcal{R}=\operatorname{diag}([0.026,0.312,0.022,0.025,0.046]) .
$$

The residence times represent the activity level of the system compartments - the smaller the residence times, the more active the compartments. The diagonal entries are ordered as

$$
r_{3}<r_{4}<r_{1}<r_{5}<r_{2} .
$$

The detritus is the least active compartment of the system in terms of energy transfer with residence time of $r_{2}=0.312 \mathrm{y}$, then comes the carnivores with $r_{5}=0.046 \mathrm{y}$. The energy transfer activity levels of the plants, detritus feeders, and bacteria are close to each other. The most active species in the Cone Spring ecosystem is the bacteria with $r_{3}=0.022 \mathrm{y}$. Therefore, the total energy storages in the least and most active compartments, the detritus and the bacteria, are completely transmitted to the system and environment in $r_{2}=113.8$ days and $r_{3}=8.2$ days, respectively.

The output-oriented subthroughflow, substorage, and diact flow and storage matrices for the Cone Spring ecosystem are analyzed by [14]. The output-oriented simple acyclic and cycling effect measures are presented below. Similar to the relationship between input-oriented simple acyclic and cycling effect measures, $\tilde{\mathrm{A}}^{\tau}$ and $\tilde{\mathrm{C}}^{\tau}$, the non-diagonal entries of $\tilde{\overline{\mathrm{A}}}^{\tau}$ are greater than the corresponding entries of $\tilde{\overline{\mathrm{C}}}^{\tau}$ for each subcompartment:

$$
\begin{gathered}
\tilde{\tilde{\mathrm{C}}}^{\tau}=\left[\begin{array}{ccccc}
0 & 0 & 0 & 0 & 0 \\
0 & 0.0268 & 0.0238 & 0.0122 & 0.0014 \\
0 & 0.0032 & 0.0195 & 0.0017 & 0.0002 \\
0 & 0.0002 & 0.0002 & 0.0023 & 0.0003 \\
0 & 0.0 & 0.0 & 0.0 & 0.0001
\end{array}\right] \text { and } \\
\tilde{\overline{\mathrm{A}}}^{\tau}=\left[\begin{array}{ccccc}
0 & 0.121 & 0.108 & 0.055 & 0.006 \\
0 & 0 & 0.115 & 0.059 & 0.007 \\
0 & 0.019 & 0 & 0.010 & 0.001 \\
0 & 0.005 & 0.004 & 0 & 0.007 \\
0 & 0.002 & 0.002 & 0.001 & 0
\end{array}\right]
\end{gathered}
$$

Note that, although the input- and output-oriented simple cycling effect matrix measures are different, the corresponding compartmental vector measures are the same as shown in Section 2.9. That is,

$$
\check{\tilde{\mathrm{C}}}^{\tau}=\tilde{\mathrm{C}}^{\tau} \mathbf{1}=\tilde{\overline{\mathrm{C}}}^{\tau} \mathbf{1}=\check{\overline{\mathrm{C}}}^{\tau}=[0,0.064,0.025,0.003,0.000]^{T} .
$$

To emphasize the difference in the interpretations of the input- and outputoriented system analysis tools, two flow-based simple acyclic effect indices, $\mathrm{a}_{3_{2}}^{\tau}$ and 


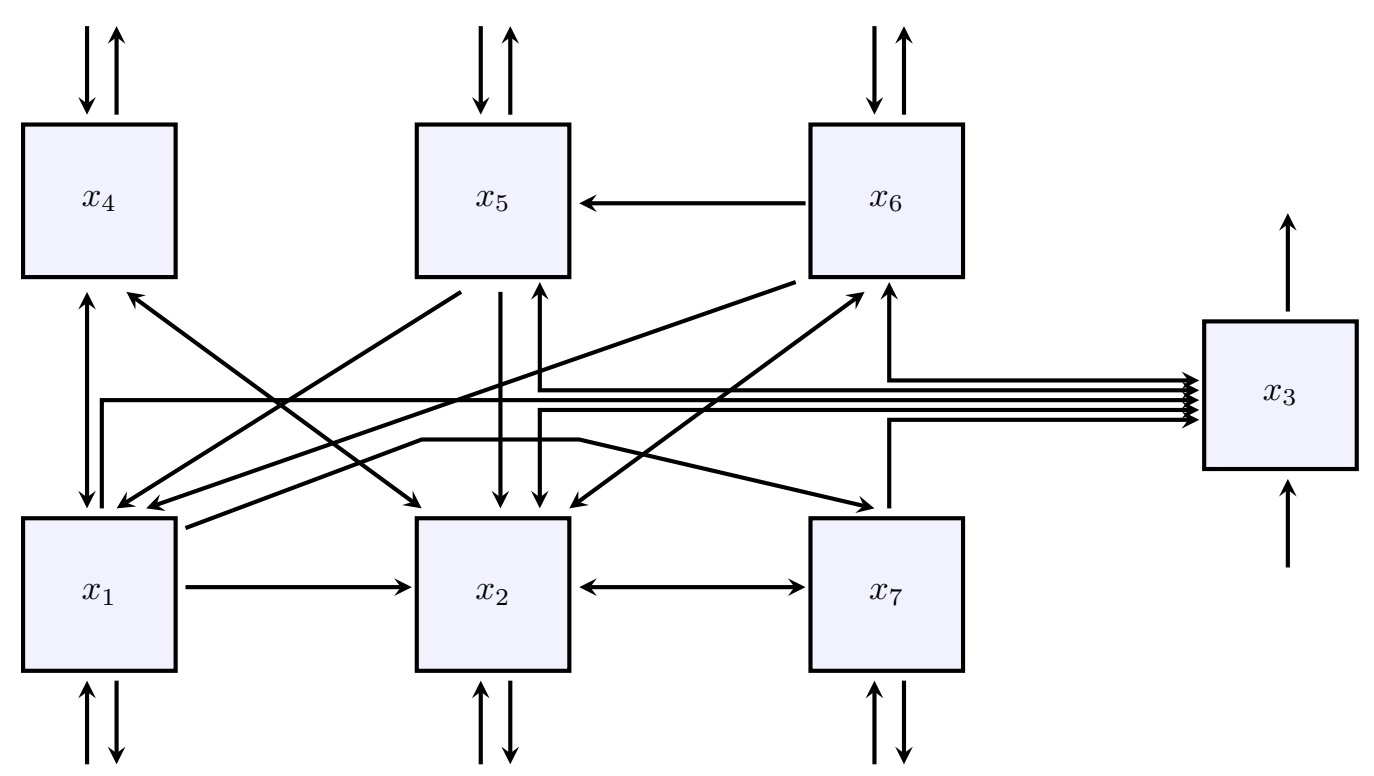

FIG. 5. Schematic representation of the model network (Case Study 3.2).

$\overline{\mathrm{a}}_{2_{3}}^{\tau}$, will be compared: The acyclic indices, $\mathrm{a}_{3_{2}}^{\tau}=0.0097$ and $\overline{\mathrm{a}}_{2_{3}}^{\tau}=0.115$, indicate that $0.97 \%$ of $\sigma^{\tau}$ is generated by $z_{2}$ at the bacteria via the detritus and $11.5 \%$ of $\sigma^{\tau}$ from the detritus is destined to contribute to $y_{3}$ via the bacteria, and these flow segments in either orientation do not cycle in the bacteria after being transmitted from the detritus.

All the other output-oriented diact measures and indices can be computed as formulated in Sections 2.9, and interpreted for various aspects of the Cone Spring ecosystem similar to their input-oriented counterparts.

3.2. Case study. The Neuse River Estuary is a drowned river valley located at the transition from the Neuse River to Pamlico Sound in North Carolina. In 1997, the State of North Carolina legislated a reduction in nitrogen loading to the estuary. As part of the monitoring program to study the estuary's response to new environmental management, nitrogen loading data is constructed for 16 seasons starting from Spring 1985 to Winter 1989 [10].

The ecosystem is modeled with seven compartments: phytoplankton particulate nitrogen, 1-PN-phyto; heterotroph particulate nitrogen, 2-PN-hetero; sediment particulate nitrogen, 3-N-sed; dissolved organic nitrogen, 4-DON; nitrate and nitrites, 5-NOx; ammonium, 6-NH4; and abiotic particulate nitrogen, 7-PN-abiotic. The compartments will be indexed in the given order; for example, $x_{1}$ represents the nitrogen storage in PN-phyto. The units for nitrogen storage and flow are $\left(\mathrm{mmol} \mathrm{m}^{-2}\right)$ and $\left(\mathrm{mmol} \mathrm{m} \mathrm{m}^{-2}\right.$ season $\left.^{-1}\right)$, respectively. The conserved quantity of interest in this case is nitrogen. Each season is considered to be a discrete time step; for example, $t=1$ corresponds to Spring 1985 and $t=16$ to Winter 1989. The system, with these seven compartments $(n=7), 22$ intercompartmental flows, environmental inputs, and outputs, is at steady state at each time step. The details of the rules and procedures for converting field data to flows and standing stocks needed for network construction are given by [10] (see Fig. 5).

The Neuse River Estuary ecosystem was recently analyzed through the proposed 

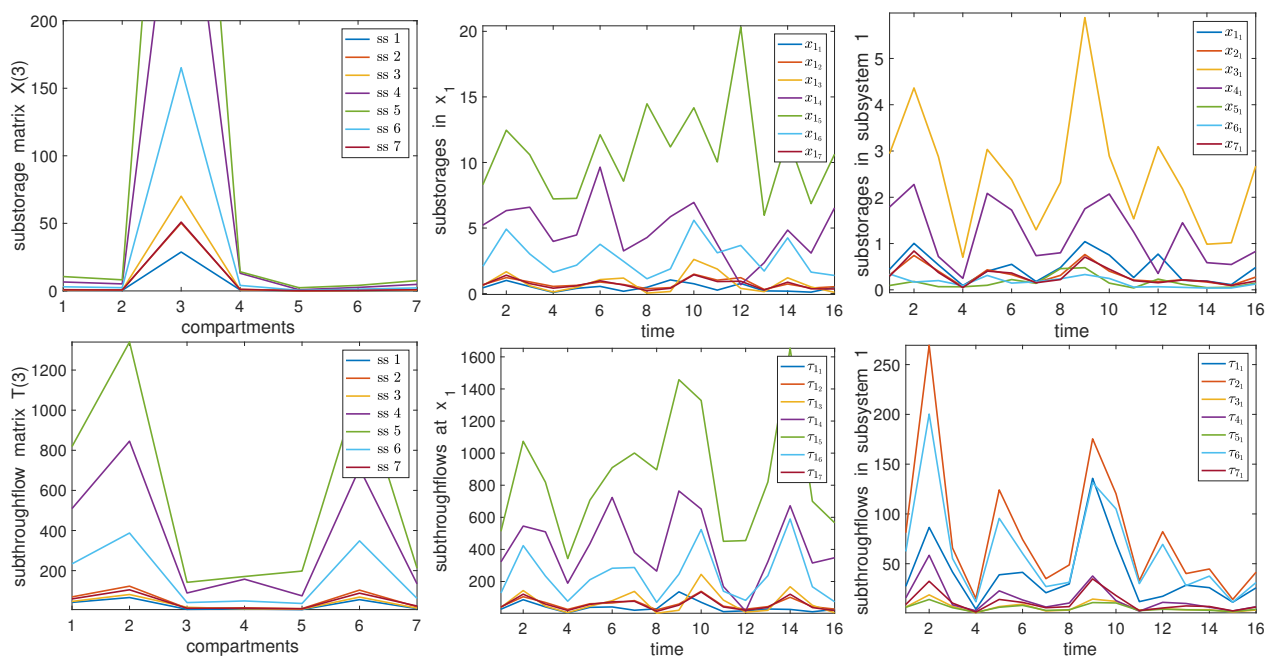

FIG. 6. The numerical results for selected elements of the substorage and subthroughflow matrix measures, $X$ and $T$. The legends (ss $1-7)$ in the first figures of each row are the subsystem numbers (column numbers of the matrices). In the matrix of $2 \times 3$ figure boxes, in the figure at $(1,3)-$ position (the substorages in subsystem 1), $x_{3_{1}}$ is scaled down by a factor of $10^{-1}$ for clarity of the presentation (Case Study 3.2).

methodology using a quasi-steady-state approach by [14]. That is, the system is analyzed at each data time step for each season under the steady-state assumption, and then, the results are combined to get the impression of potential dynamic behavior. The substorage and subthroughflow matrix measures and the transient and diact flows and storages are presented for this ecosystem model. In this case study, the diact effect, utility, and residence time measures and indices together with their ecological implications are presented for this model. The model is also studied extensively in the literature, but some important results obtained through the proposed methodology have not been observed in these analyses or could not be demonstrated, although anticipated, as shown further below [10, 6, 49, 59].

The system partitioning methodology yields the subthroughflow and substorage matrix measures that quantify the distribution of environmental inputs and the organization associated storages within the system. The compartment-wise and time-wise cross sections of the system are presented in Fig. 6. The substorage and subthroughflow matrices at time $t=3$ (Fall 1985), $X(3)$ and $T(3)$, are presented. The substorages and subthroughflows at a specific compartment, $x_{1}$, are also presented for a typical compartmental behavior. Moreover, the substorage and subthroughflow vectors for the first subsystem, $\left[x_{1_{1}}, x_{2_{1}} \ldots, x_{7_{1}}\right]$ and $\left[\tau_{1_{1}}, \tau_{2_{1}} \ldots, \tau_{7_{1}}\right]$, which are derived from the environmental input into the first compartment (PN-phyto), $z_{1}$, are also depicted in Fig. 6 .

In general terms, $x_{i}$ is the composite nitrogen storage in compartment $i$ of the original system. It cannot be used to distinguish the nitrogen derived from individual environmental inputs. On the other hand, $x_{i_{k}}$ is the nitrogen storage in compartment $i$ that is generated by individual environmental input $z_{k}$. Therefore, the system partitioning methodology allows for partitioning composite compartmental nitrogen into subcompartmental nitrogen segments based on their constituent environmental sources. In other words, the system partitioning enables tracking the fate of environ- 

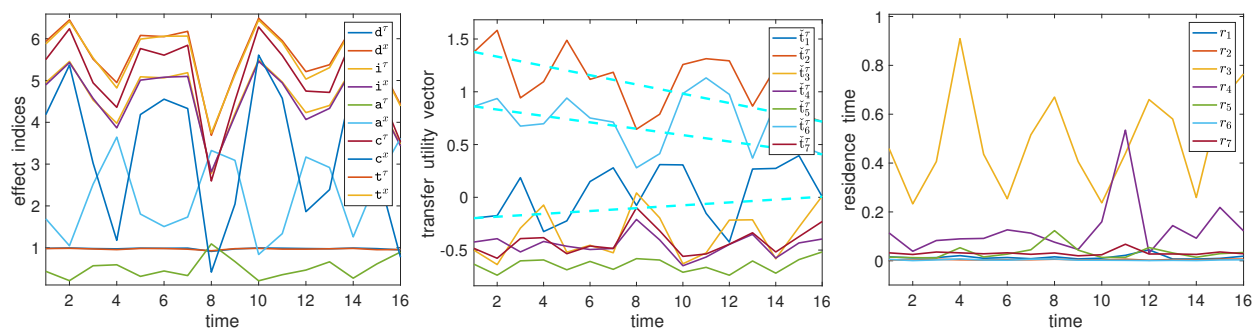

FIG. 7. The diact effect indices, transfer utility vector $\check{\mathbb{t}}^{\tau}$ (in the figure legend, $\check{\mathrm{t}}^{\tau}$ notation is used instead, due to the limited font library of Matlab software), and residence times against time. The residence time for the third compartment, $r_{3}$, is scaled down by a factor of $10^{-1}$ for clarity of the presentation (Case Study 3.2).

mental nitrogen inputs individually and separately within the system. The subsystem partitioning methodology then enables tracking the fate of arbitrary intercompartmental nitrogen flows and associated nitrogen storages within the subsystems. Therefore, the effect of one compartment on another in terms of not only direct but also indirect nitrogen transactions can be determined. Parallel interpretations are also valid for the nitrogen throughflows of the original system, $\tau_{i}$, and the subthroughflows of the decomposed system, $\tau_{i_{k}}$. These interpretations can be extended further to the output-oriented system and subsystem partitioning as well.

The subsystem partitioning methodology construct a platform for the definition of the diact flows and associated storages based on which the diact effect and utility measures and indices are formulated in the present manuscript. It was reported by [10] that indirect flows tend to dominate direct flows in the system. This dominance is apparent in the given order of the effect indices presented in Fig. 7:

$$
\mathrm{t}^{x} \approx \mathrm{t}^{\tau}>\mathrm{i}^{x} \approx \mathrm{i}^{\tau}>1>\mathrm{d}^{x} \approx \mathrm{d}^{\tau} .
$$

Therefore, the method outcomes match the ecological insights about the Neuse River Estuary ecosystem. As outlined in the Cone Spring ecosystem analysis in the previous section, the dominance of a certain diact transaction type in the system can be used as an indicator for ecosystem characterization.

Biological activities increase in springs and summers and slow down during winters. Physical processes such as river discharge and nutrient loading tend to be high and flushing times (time required for river flow to replace freshwater in the estuary) short during colder months [9]. Therefore, it is anticipated that the proportion of indirect flows varies seasonally, contributing more in the spring and less in the winter. There are attempts in the literature to demonstrate the seasonality in indirect effects that failed, possibly due to the authors' indirect effect formulations as discussed in Sections 2.2 and $2.6[39,41,6,4,33]$. The proposed indirect effect indices can clearly capture the temporal system behavior as anticipated (see Fig. 7).

Interestingly, both flow- and storage-based cycling and acyclic effect indices are oscillating in opposite phases and not well-ordered, as presented in Fig. 7. As a matter of fact, the acyclic effect indices oscillate in opposite phase to all the other diact effect indices. Considering the supplementary nature of the acyclic and cycling flows introduced by [14], we hypothesize that, possibly due to the slower biological activities during the winters, the transfer flows fall short of completing the nitrogen cycle and, therefore, the cycling flows decrease and the acyclic flows increase within the ecosystem during the winters. These results and interpretations are also consistent 
with the periodic behaviors of the acyclic efficiencies introduced by [12]. These conclusions imply that such precise quantitative analysis of ecosystems may lead to more ecological theoretical developments.

During the course of this four-year experiment, there is a gradual decrease in normalized relative net nitrogen inflow transmitted directly or indirectly from all system compartments to the heterotrophs (2-PN-hetero) and ammonium (6-NH4) but a gradual increase in that to the phytoplankton (1-PN-phyto), as indicated by transfer utility vector graphs, $\dot{\mathbb{t}}^{\tau}(t)$, in Fig. 7 . To our knowledge, this overall tendency was not detected by previous studies of this data in the literature either. This outcome potentially explains the high phytoplankton production and biomass, harmful algal blooms that the Neuse River and its estuary have been experiencing [10].

In accordance with this result, the residence time graphs show that the compartments with the smallest residence time are the heterotrophs (2-PN-hetero) and ammonium (6-NH4) with about 0.1 year residence time. Therefore, they are the most active compartments in terms of the nitrogen transfer. The seasonality in the residence time of particulate nitrogen in the sediments $(3-\mathrm{N}$-sed) is more pronounced. The nitrogen residence time increases to 6-9 years in the winters, likely due to slow biological activities, from about 2 years in the summers (see Fig. 7).

The results presented above indicate that the proposed methodology brings out ecological inferences about the system that are not available through analysis of the original system by the state-of-the-art techniques.

4. Discussion. Nature is constantly changing as ecosystems evolve and adapt to various factors. Yet, current methodologies for ecosystem analysis are designed mainly for special cases, such as linear models and static systems. Effective methods for analyzing various aspects of dynamic nonlinear ecosystem models are imperative.

Despite the past decades' numerous attempts at developing methods for analyzing dynamic systems, systematic treatments of nonlinear compartmental models within an analytical framework have not emerged yet. A novel mathematical theory and method for analysis of nonlinear dynamical compartmental systems has recently been introduced by $[13,11]$. The static version of this methodology is developed in a separate work [14]. This comprehensive approach is based on analytical and explicit, mutually exclusive and exhaustive, novel system and subsystem partitioning methodologies. The system partitioning refines system analysis from the current compartmental level to the subcompartmental level. While the system partitioning determines the distribution of environmental inputs and the organization of the associated storages generated by these inputs within the system, the subsystem partitioning ascertains the distribution of intercompartmental flows and the organization of associated storages within the subsystems. Therefore, the mathematical method as a whole decomposes the system flows and storages to the utmost level. The substorage and subthroughflow matrices, as well as the transient and static direct, indirect, cycling, acyclic, and transfer (diact) flows and storages are formulated based on these methodologies by [14].

Considering a hypothetical ecosystem modeling a food web with several interacting species for which the effect of a specific poison needs to be investigated, one of the most critical inquiries would be about the fate of the poison within the system. Current mathematical methods for static models cannot track the fate of the toxin, that is, cannot determine the amount of toxin traveling throughout the web and the associated storages generated by these flows. The proposed methodology enables monitoring how an arbitrary amount of the toxin would spread throughout 
the food web and, therefore, ascertaining the effect of the toxin in one species on any other in the web. Direct effects are relatively easier to analyze through direct interspecific interactions, and the current techniques mainly focus on such analysis. On the other hand, the transfer (total) effect of the toxin in one species, directly or indirectly through other species, on another has never been formulated before. The transfer effect is one of the multiple quantitative system analysis tools introduced in the present manuscript.

The current measures and indices have significant shortcomings as well. For example, Finn's flow-based cycling index, FCI, was developed four decades ago, and has been an essential measure for ecosystem analysis but only for systems at steady state $[20,21]$. The storage-based cycling effect index, SCI, was recently introduced by [34], also for static systems. Moreover, various versions of the static flow-based indirect effect indices have been formulated by several groups, but none of these seem to be precisely identifying the indirect effect behavior, as discussed in Section 2.6 $[39,41,54,4,33]$. The flow- and storage-based dynamic cycling and indirect effect indices were introduced recently by [12] at both compartmental and subcompartmental levels. Their static versions are also formulated in the present manuscript. Although derived with a different rationale, the proposed input-oriented, compartmental, flowand storage-based simple cycling effect indices are equivalent to the FCI and SCI, respectively. The proposed indirect effect indices ascertain the corresponding phenomena more precisely than the previous static formulations, as demonstrated in Section 3.

The static methodology proposed by [14] introduces multiple new mathematical system analysis tools. The subthroughflow and substorage matrix measures, for example, monitor the distribution of environmental inputs and the organization of the associated storages generated by the inputs individually and separately, as introduced by [14]. The transient and static diact transaction types introduced in the same article enable the determination of the flows and storages transmitted along a given flow path or from one compartment, directly or indirectly, to any other within the system. This static methodology allows also for both input- and output-oriented analyses of static ecosystems through the system partitioning methodology based on environmental inputs and outputs, respectively. The duality of the input- and output-oriented analyses is demonstrated through novel similarity relationships by [14].

The proposed methodology also constructs a base for the development of new mathematical system analysis tools as quantitative ecological indicators. Based on the proposed method, multiple new measures and indices of matrix, vector, and scalar type for the input- and output-oriented, flow- and storage-based, simple and composite diact effects, utilities, and residence times are systematically formulated at both compartmental and subcompartmental levels in the present manuscript. Except the simple cycling and indirect effect indices as outlined above, all of these measures and indices are introduced analytically and explicitly for the first time in literature. These system analysis tools are the static versions of the dynamic measures and indices introduced by [12].

The input- and output-oriented diact effect measures and indices quantify the influence of compartments directly or indirectly on the others. The input and outputoriented diact utility measures and indices then determine the relative influence of compartments on each other. The residence time matrix is another novel system measure proposed in the present manuscript which ascertains the compartmental activity levels. Several case studies presented in Section 3 illustrate the efficiency and wide applicability of the proposed measures and indices, and demonstrate that these 
mathematical system analysis tools can be used for rigorous analysis of ecosystems as quantitative ecological indicators.

The diact effect and utility measures quantify the diact interactions between any two compartments of the system, in terms of flow and storage transfer. The precise measurements of the diact intercompartmental effects and utilities enable the analysis of interspecific interactions within food webs. A quantitative technique for the classification and characterization of the main interspecific interaction types and the determination of their strength is also developed based on the diact effects and utility measures in the present manuscript.

The present work is the first comprehensive approach in literature that leads to a holistic methodology for ecosystem analysis as outlined in Section 2.4. The current static measures and indices are reformulated with a different derivation rationale in the context of the proposed methodology and, therefore, are integrated with all the other novel system analysis tools introduced in the present work. Moreover, unique relationships among some current static measures are unveiled through the proposed methodology. Corrections in some current formulations are also suggested. The cumulative storage and throughflow distribution matrices, $S$ and $N$, have been considered unrelated and treated separately in the literature; however, it is shown that they are related by a factor of the residence time matrix, $\mathcal{R}$. The input- and output-oriented vector and matrix measures are also effectively integrated through the residence time matrix. Therefore, $\mathcal{R}$ has a central role in connecting various measures and integrating different components of the proposed methodology. Also, the duality of input- and output-oriented system analyses establishes new similarity relationships between the cumulative storage and throughflow distribution matrices and integrates the system analyses in both orientations. A number of equivalent formulations for $S$ and $N$ are presented, which combines multiple matrix measures all together. The system and subsystem partitioning methodologies are also integrated through the geometric series expansions of the distribution matrices. Consequently, various components of ecosystem analysis are effectively combined and holistically integrated within the proposed unifying mathematical framework.

5. Conclusions. In the present manuscript, we systematically formulated multiple measures and indices of matrix, vector, and scalar type for the holistic analysis of static ecosystems. These input- and output-oriented measures and indices for the diact effects, utilities, and residence times are novel mathematical system analysis tools that serve as quantitative ecological indicators. A quantitative technique for the characterization and classification of main interspecific interaction types and the determination of their strength within food webs is also developed based on the diact effect and utility measures.

The proposed static system measures and indices extract detailed information about ecosystems' characteristics, functions, and behaviors. As quantitative system analysis tools, they monitor the flow distribution and storage organization, quantify the effect and utility of one compartment directly or indirectly on another, determine the residence times and compartmental activity levels within ecosystems. Several case studies from ecosystem ecology are presented to demonstrate the efficiency and wide applicability of the proposed measures and indices.

The proposed methodology extends the strength and applicability of the stateof-the-art techniques and provides significant advancements in theory, methodology, and practicality. It serves, therefore, as a quantitative platform for testing empirical hypotheses, ecological inferences, and, potentially, theoretical developments. 
Acknowledgments. The author would like to thank Hasan Coskun for useful discussions and his helpful comments that improved the manuscript.

\section{REFERENCES}

[1] T. Allen and M. Giampietro, Holons, creaons, genons, environs, in hierarchy theory: Where we have gone, Ecological Modelling, 293 (2014), pp. 31-41, https://doi.org/10.1016/j. ecolmodel.2014.06.017.

[2] R. Bailey, J. K. Allen, And B. Bras, Applying ecological input-output flow analysis to material flows in industrial systems: Part $i$ : Tracing flows, Journal of Industrial Ecology, 8 (2004), pp. 45-68, https://doi.org/10.1162/1088198041269346.

[3] A. Belgrano, U. M. Scharler, J. Dunne, and R. E. Ulanowicz, Aquatic Food Webs: An Ecosystem Approach, Oxford University Press, Oxford, U.K, 2005.

[4] S. R. Borrett, M. A. Freeze, And A. K. Salas, Equivalence of the realized input and output oriented indirect effects metrics in Ecological Network Analysis, Ecological Modelling, 222 (2011), pp. 2142-2148, https://doi.org/10.1016/j.ecolmodel.2011.04.003, https: //arxiv.org/abs/1103.6276.

[5] S. R. Borrett And M. K. LaU, enaR : An $R$ package for ecosystem network analysis, Methods in Ecology and Evolution, 5 (2014), pp. 1206-1213, https://doi.org/10.1111/2041-210x. 12282.

[6] S. R. Borrett, S. J. Whipple, B. C. Patten, and R. R. Christian, Indirect effects and distributed control in ecosystems: Temporal variation of indirect effects in a sevencompartment model of nitrogen flow in the neuse river estuary, usa - time series analysis, Ecological Modelling, 194 (2006), pp. 178-188, https://doi.org/https://doi.org/10.1016/j. ecolmodel.2005.10.011.

[7] B. C. Patten and S. J. Whipple, Ecological Utility Analysis: Determination of Interaction Types Between Organisms in Ecosystems, vol. 2, WIT Press, 09 2011, https://doi.org/10. 2495/ECO-V2-N2-88-96.

[8] V. Christensen and D. PAUly, ECOPATH II-a software for balancing steady-state ecosystem models and calculating network characteristics, Ecological modelling, 61 (1992), pp. 169185.

[9] R. R. Christian, J. N. Boyer, and D. W. Stanley, Multi-year distribution patterns of nutrients within the neuse river estuary, north carolina, Marine Ecology Progress Series, 71 (1991), pp. 259-274.

[10] R. R. Christian and C. R. Thomas, Neuse river estuary modeling and monitoring project stage 1: Network analysis for evaluating the consequences of nitrogen loading, Tech. Report UNC-WRRI-2000-325-F, Biology Department, East Carolina University, 2000.

[11] H. Coskun, Dynamic ecological system analysis, Heliyon, 5 (2019), https://doi.org/10.1016/j. heliyon.2019.e02347, https://doi.org/10.31219/osf.io/35xkb.

[12] H. Coskun, Dynamic ecological system measures, Results in Applied Mathematics, (2019), https://doi.org/10.1016/j.rinam.2019.100007, https://doi.org/10.31219/osf.io/j2pd3.

[13] H. Coskun, Nonlinear decomposition principle and fundamental matrix solutions for dynamic compartmental systems, Discrete and Continuous Dynamical Systems - B, (2019), https: //doi.org/10.3934/dcdsb.2019155, https://doi.org/10.31219/osf.io/cyrzf.

[14] H. Coskun, Static ecological system analysis, Theoretical Ecology, (2019), https://doi.org/10. 1007/s12080-019-0421-8, https://doi.org/10.31219/osf.io/zqxc5.

[15] D. DeAngelis, Energy flow, nutrient cycling, and ecosystem resilience, Ecology, 61 (1980), pp. $764-771$.

[16] B. D. FATH, Network mutualism: Positive community-level relations in ecosystems, Ecological Modelling, 208 (2007), pp. 56-67, https://doi.org/10.1016/j.ecolmodel.2007.04.021.

[17] B. D. Fath AND S. R. BorRett, A Matlab® function for Network Environ Analysis, Environmental Modelling and Software, 21 (2006), pp. 375-405, https://doi.org/10.1016/j.envsoft. 2004.11.007.

[18] B. D. Fath and B. C. Patten, Network synergism: Emergence of positive relations in ecological systems, Ecological Modelling, 107 (1998), pp. 127-143, https://doi.org/10.1016/ S0304-3800(97)00213-5.

[19] B. D. Fath And B. C. Patten, Review of Network the Foundations of Environ Analysis, Ecosystems, 2 (1999), pp. 167-179.

[20] J. Finn, Measures of structure and functioning derived from analysis of flows, Journal of Theoretical Biology, 56 (1976), pp. 363-380.

[21] J. T. Finn, Flow Analysis of Models of the Hubbard Brook Ecosystem, Ecology, 61 (1980), 
pp. $562-571$.

[22] B. Hannon, The structure of ecosystems, Journal of theoretical biology, 41 (1973), pp. 535-546.

[23] M. Higashi and B. C. Patten, Dominance of indirect causality in ecosystems, The American Naturalist, 133 (1989), pp. 288-302.

[24] H. HiRATA AND R. E. UlanOwicz, Information theoretical analysis of the aggregation and hierarchical structure of ecological networks, Journal of Theoretical Biology, 116 (1985), pp. 321-341.

[25] R. Holt, Community modules, in Multitrophic Interactions in Terrestrial Ecosystems, A. Gange and V. Brown, eds., 36th Symposium, British Ecological Society, Blackwell Science, 1997, pp. 333-349.

[26] R. D. Holt, Predation, apparent competition, and the structure of prey communities, Theoretical Population Biology, 12 (1977), pp. 197-229, https://doi.org/10.1016/0040-5809(77) 90042-9, https://arxiv.org/abs/0040580977900429.

[27] C. KAZANCI, Network calculations II: a user's manual for EcoNet, in Handbook of Ecological Modelling and Informatics, WITPRESS LTD., 2009, pp. 325-350, https://doi.org/10.2495/ 978-1-84564-207-5/18.

[28] C. KaZAnci AND M. R. Adams, Ecological utility theory: Solving a series convergence issue, Ecological Modelling, 358 (2017), pp. 19-24, https://doi.org/https://doi. org/10.1016/j.ecolmodel.2017.04.016, http://www.sciencedirect.com/science/article/pii/ S0304380016307049.

[29] W. W. Leontief, Quantitative input and output relations in the economic systems of the united states, The review of economic statistics, 18 (1936), pp. 105-125.

[30] W. W. Leontief, Input-output economics, Oxford University Press on Demand, New York, 1986.

[31] R. L. Lindeman, The trophic-dynamic aspect of ecology, Ecology, 23 (1942), pp. 399-417.

[32] C. Loenle, Indirect effects: A critique and alternate methods, Ecology, 71 (1990), pp. 23822386, https://doi.org/10.2307/1938651, https://doi.org/10.2307/1938651.

[33] Q. Ma AND C. KazANCI, Analysis of indirect effects within ecosystem models using pathwaybased methodology, Ecological Modelling, 252 (2013), pp. 238-245, https://doi.org/10. 1016/j.ecolmodel.2012.05.002.

[34] Q. Ma AND C. KAZANCI, How much of the storage in the ecosystem is due to cycling?, Journal of Theoretical Biology, 357 (2014), pp. 134-142, https://doi.org/10.1016/j.jtbi.2014.05.014.

[35] J. Matis And B. Patten, Environ analysis of linear compartmental systems: the static, time invariant case, Statistical Ecology, 48 (1981), pp. 527-565.

[36] A. B. Menge, Detection of direct versus indirect effects: were experiments long enough?, AMERICAN NATURALIST, 149 (1997), pp. 801-823.

[37] B. A. Menge, Indirect effects in marine rocky intertidal interaction webs: Patterns and importance, Ecological Monographs, 65 (1995), pp. 21-74, https://doi.org/10.2307/2937158, https://doi.org/10.2307/2937158.

[38] R. T. PAINE, Food web complexity and species diversity, American Naturalist, 100 (1966), pp. $65-75$.

[39] B. PATten, Energy cycling, length of food chains, and direct versus indirect effects in ecosystems, Canadian Bulletin of Fisheries and Aquatic Sciences, 213 (1985), pp. 119-138.

[40] B. C. PAtten, Systems approach to the concept of environment, Ohio Journal of Science, 78 (1978), pp. 206-222.

[41] B. C. Patten, Energy cycling in the ecosystem, Ecological Modelling, 28 (1985), pp. 1-71, https://doi.org/10.1016/0304-3800(85)90013-4.

[42] B. C. Patten, Environ theory and indirect effects: A reply to loehle, Ecology, 71 (1990), pp. 2386-2393, https://doi.org/10.2307/1938652, https://doi.org/10.2307/1938652.

[43] B. C. Patten, Network ecology: Indirect determination of the life-environment relationship in ecosystems, in Theoretical Studies of Ecosystems: The Network Perspective, M. Higashi and T. P. Burns, eds., Cambridge University Press, 1991, pp. 288-351.

[44] B. C. Patten, Energy, emergy and environs, Ecological Modelling, 62 (1992), pp. 29-69, https://doi.org/10.1016/0304-3800(92)90081-O.

[45] B. C. Patten, R. W. Bosserman, J. T. Finn, and W. G. Cale, Propagation of cause in ecosystems, Systems analysis and simulation in ecology, 4 (1976), pp. 457-579.

[46] B. C. Patten and M. Higashi, Modified cycling index for ecological applications, Ecological Modelling, 25 (1984), pp. 69-83.

[47] R. Pilette, Evaluating direct and indirect effects in ecosystems, The American Naturalist, 133 (1989), pp. 303-307, http://www.jstor.org/stable/2462307.

[48] M. Rasmussen, A. Hastings, M. J. Smith, F. B. Agusto, B. M. Chen-Charpentier, F. M. Hoffman, J. Jiang, K. E. O. Todd-Brown, Y. Wang, Y.-P. Wang, and Y. Luo, 
Transit times and mean ages for nonautonomous and autonomous compartmental systems, Journal of Mathematical Biology, 73 (2016), pp. 1379-1398, https://doi.org/10.1007/ s00285-016-0990-8, https://doi.org/10.1007/s00285-016-0990-8.

[49] J. R. Schramski, D. K. Gattie, B. C. Patten, S. R. Borrett, B. D. Fath, and S. J. WhIPPLE, Indirect effects and distributed control in ecosystems: Distributed control in the environ networks of a seven-compartment model of nitrogen flow in the Neuse River Estuary, USA-Time series analysis, Ecological Modelling, 206 (2007), pp. 18-30, https: //doi.org/10.1016/j.ecolmodel.2007.03.023.

[50] J. R. Schramski, C. Kazanci, And E. W. Tollner, Network environ theory, simulation, and EcoNetß, 2.0, Environmental Modelling and Software, 26 (2011), pp. 419-428, https: //doi.org/10.1016/j.envsoft.2010.10.003.

[51] S. Y. StRAuss, Indirect effects in community ecology: Their definition, study and importance., Trends in ecology \& evolution (Personal edition), 6 (1991), pp. 206-210, https://doi.org/ 10.1016/0169-5347(91)90023-Q.

[52] L. J. Tilly, The structure and dynamics of Cone Spring, Ecological Monographs, 38 (1968), pp. 169-197.

[53] L. K. Tuominen, S. J. Whipple, B. C. Patten, Z. Y. Karatas, and C. Kazanci, Contribution of throughflows to the ecological interpretation of integral network utility, Ecological Modelling, 293 (2014), pp. 187-201, https://doi.org/10.1016/j.ecolmodel.2014.01.027.

[54] R. Ulanowicz and C. Puccia, Mixed trophic impacts in ecosystems., Coenoses, 5 (1990), pp. $7-16$.

[55] R. E. Ulanowicz, Mass and energy flow in closed ecosystems, Journal of Theoretical Biology, 34 (1972), pp. 239-253, https://doi.org/10.1016/0022-5193(72)90158-0.

[56] R. E. Ulanowicz, Quantitative methods for ecological network analysis, Computational Biology and Chemistry, 28 (2004), pp. 321-339, https://doi.org/10.1016/j.compbiolchem.2004. 09.001.

[57] R. E. Ulanowicz, R. D. Holt, and M. Barfield, Limits on ecosystem trophic complexity: insights from ecological network analysis, Ecology Letters, 17 (2013), pp. 127-136, https: //doi.org/10.1111/ele.12216.

[58] R. E. Ulanowicz AND J. J. KaY, A package for the analysis of ecosystem flow networks, Environmental Software, 6 (1991), pp. 131-142, https://doi.org/10.1016/0266-9838(91) 90024-K.

[59] S. J. Whipple, B. C. Patten, and S. R. Borrett, Indirect effects and distributed control in ecosystems: Comparative network environ analysis of a seven-compartment model of nitrogen storage in the Neuse River Estuary, USA: Time series analysis, Ecological Modelling, 293 (2014), pp. 161-186.

[60] J. Wootton, Indirect effects in complex ecosystems: recent progress and future challenges, Journal of Sea Research, 48 (2002), pp. 157-172, https://doi.org/10.1016/S1385-1101(02) 00149-1.

[61] J. T. Wоotton, Indirect effects and habitat use in an intertidal community: Interaction chains and interaction modifications, The American Naturalist, 141 (1993), pp. 71-89, https://doi.org/10.1086/285461, https://doi.org/10.1086/285461.

[62] J. T. Wootton, The nature and consequences of indirect effects in ecological communities, Annual Review of Ecology and Systematics, 25 (1994), pp. 443-466, https://doi.org/10. 1146/annurev.es.25.110194.002303, https://doi.org/10.1146/annurev.es.25.110194.002303. 